\title{
Recent Developments in Econometric Modeling and Forecasting
}

\author{
GANG LI $^{a}$, HAIYAN SONG ${ }^{b}$ and STEPHEN F. WITT ${ }^{a *}$ \\ ${ }^{a}$ School of Management, University of Surrey, Guildford GU2 7XH, United Kingdom \\ ${ }^{b}$ School of Hotel and Tourism Management, The Hong Kong Polytechnic University, \\ Hung Hom, Kowloon, Hong Kong
}

Eighty-four post-1990 empirical studies of international tourism demand modeling and forecasting using econometric approaches are reviewed. New developments are identified and it is shown that applications of advanced econometric methods improve the understanding of international tourism demand. An examination of the 22 studies which compare forecasting performance suggests that no single forecasting method can outperform the alternatives in all cases. However, the timevarying parameter (TVP) model and structural time series model with causal variables perform consistently well.

Keywords: review, tourism demand, modeling, forecasting

\section{INTRODUCTION}

The rapid expansion of international tourism has motivated growing interest in tourism demand studies. The earliest work of can be traced back to the 1960 s, notably pioneered by Guthrie (1961), followed by Gerakis (1965) and Gray (1966). The last four decades have seen great developments in tourism demand analysis, in terms of the diversity of research interests, the depth of theoretical foundations, and advances in research methodologies. Modeling tourism demand in order to analyze the effects of various determinants, and accurate forecasting of future tourism demand, are two major focuses of tourism demand studies. The developments in tourism forecasting methodologies fall into several streams, amongst which the econometric approach plays a very important role in tourism demand studies. This methodology is able to interpret the causes of variations of tourism demand, support policy evaluation and strategy making, and predict future trends in tourism development.

Since the beginning of the 1960s a large number of empirical studies on tourism demand have been published. Crouch (1994c) carried out an extensive literature search and found over 300 publications during the period 1961-1993. Since then about 120 papers on tourism demand modeling and/or forecasting have been added to the tourism demand literature. A comprehensive overview of the existing empirical work will "provide guidance to other researchers interested in undertaking other similar studies" (Crouch 1994c, p. 12). A number of review papers have been published.

\footnotetext{
* Corresponding author.
} 
Crouch (1994a, 1994b, 1994c, 1994d, 1995, 1996) examined about 80 econometric studies of international tourism demand covering the period 1961-1993. Using metaanalysis techniques, Crouch (1994a, 1994b, 1995, 1996) identified various inter-study differences that explained the variations in the findings, principally with respect to demand elasticities. Lim (1997a, 1997b, 1999) reviewed 100 papers on tourism demand modeling published during the period 1961-1994. Lim (1997a, 1997b) discussed the choice of dependent and explanatory variables, as well as the functional specifications and data used. Following these papers, Lim (1999) further selected 70 studies for meta-analysis. By calculating effect sizes, Lim (1999) attempted to generalise the relationships between international tourism demand and income, transportation cost and tourism prices. Unlike the abovementioned review studies, Sheldon and Var (1985), Uysal and Crompton (1985), and Witt and Witt (1995) focused on tourism demand forecasting practice. In their review, Sheldon and Var (1985) considered only 11 studies, all but one being published before 1978 . Uysal and Crompton (1985) provided an overview of various forecasting approaches applied to tourism studies, but no insight into individual studies was provided. Witt and Witt (1995) reviewed 40 empirical studies published over 3 decades but all prior to 1992.

The continuing growth of world-wide tourism demand in the 1990s stimulated stronger interest in studies in this field, particularly using the econometric approach. In Crouch's studies, 5, 33 and 42 papers were identified in the 1960s, 1970s and 1980s, respectively. Lim collected 4, 26, and 50 studies in the same time spans for her review. Since the start of the 1990s, over 80 pieces of empirical research have been found regarding econometric modeling and forecasting of tourism demand. However, very few of the latter studies have been included in the previous review. In their reviews, Lim only included 20 papers published during this period, Crouch 5 papers, and Witt and Witt 3 papers. All of these papers were published between 1990 and 1994, and no later publications have been reviewed. This study, therefore, aims to provide an up-to-date comprehensive review of recent studies on tourism demand modeling and forecasting.

It should be noted that meta-analysis is a useful methodology for reviewing literature, "which allows statistical generalizations to be made with respect to the combined evidence across studies" (Lim 1999, p. 273). A primary analysis shows that most of the general conclusions drawn by Crouch $(1994 a, 1994 b, 1995,1996)$ and Lim (1999) regarding inter-study differences (e.g. model specification, sample period and origin-destination pair concerned) accounting for the variation in demand elasticities still hold in the studies currently reviewed. Therefore, this study will not repeat these tests. Nevertheless, with a particular focus on the post-1990 publications on econometric modeling and forecasting of tourism demand, this paper will identify recent developments of tourism demand studies in terms of modeling techniques and their forecasting performance. In addition, for the first time, some findings from studies using the Almost Ideal Demand System (AIDS) models are covered in this review.

\section{DATA DESCRIPTION}

There are 81 empirical studies on econometric modeling and forecasting of tourism demand published during 1990-2004 that have been collected for review, supplemented by 3 publications from the 1980s focusing on a particular AIDS model. The literature search includes a computer search of databases of electronic literature 
and a manual search for references cited in the literature including book chapters and conference papers. The selected papers cover a wide range of journals in tourism, economics and forecasting. Annuals of Tourism Research, Journal of Travel Research, Tourism Management, Tourism Economics, Applied Economics, and the International Journal of Forecasting appear to be most frequently selected for publication of tourism demand modeling and forecasting studies. 23 studies not only model tourism demand and examined various influencing factors, but also compare the forecasting performance of alternative econometric models, with some time-series models as benchmarks. Therefore, 84 studies enter the review of tourism demand modeling and 31 of them are to be further considered for the evaluation of econometric models' forecasting performance.

\section{ECONOMETRIC MODELING OF TOURISM DEMAND}

A detailed summary of the 84 studies under review is shown in Table 1. In comparison with the earlier studies reviewed by Crouch (1994d) and Lim (1997a), some differences, as either emerging or vanishing trends, are identified.

\section{Data Types and Periods}

The data used in the reviewed studies covers the period 1960-2001. As with previous studies, three data frequencies were used in the post 1990 studies: annual, quarterly and monthly data. Although annual data still dominated the research in this period, quarterly data have been used more often, in line with the increasing interest in analysing the seasonality of international tourism demand. The spans of sample periods were 25 years, 76 quarters and 165 months on average when annual, quarterly and monthly data are concerned, respectively. Overall, they were longer than the average period of those reviewed by Crouch (1994d), which was only 14 years. The increasing number of observations results in more degrees of freedom in model estimation and gives more flexibility to consider additional influencing factors and/or extend the lag structure to capture the dynamics of tourism demand more sufficiently. Meanwhile, some advanced econometric models, which require estimation of more parameters such as the time-varying-parameter (TVP) model, are able to be introduced into tourism demand studies.

\section{Origin/Destination}

Western European and North American countries dominated tourism research studies prior to 1990 . This trend was related to their great contribution to international tourism development, as both inbound and outbound tourism in theses areas accounted for a very large proportion of global tourism flows. These areas have continued to draw a great deal of researchers' attention in the last decade. Among the 84 studies being reviewed, 59 and 50 referred to UK and US tourism, respectively, either as a tourist destination or origin country. Germany, France Spain and Italy also received considerable attention. Meanwhile, international tourism in the East Asia and Pacific region has shown the fastest growth in the last decade. Correspondingly, this region attracted more and more research interest. In particular, 30 studies are related to Japanese outbound tourism, 23 are related to Australian tourism (mainly inbound), and 8 are related to Hong Kong and Korea (inbound and outbound). The main travel routes were from the UK and the USA to Mediterranean countries, Australia and Hong Kong, and from Japan to Australia and Hong Kong. 
TABLE 1

SUMMARY OF ECONOMETRIC STUDIES ON TOURISM DEMAND MODELING AND FORECASTING

\begin{tabular}{l}
\hline \hline Legend: \\
1. Data frequency \& period \\
A: annual \\
M: monthly \\
Q: quarterly \\
2. Region focused \\
I: inbound \\
O: outbound \\
3. Dependent variable \\
-B: on business \\
-H: for holidays \\
-VFR: visiting friends \& relatives \\
BS: budget share of TE \\
EX: exports \\
IM: imports \\
ITC: No. of Inclusive tour chatters \\
NAC: No. of tourist accommodation \\
TA: tourist arrivals \\
TAHA: TA in hotels and apartments \\
TE: tourism receipts/expenditure \\
TM: tourism imports \\
TN: number of nights \\
TX: tourism exports \\
4. Independent variable \\
C: relative tourism price unadjusted by ER \\
C: tourism price in destination \\
Cir: tourism lifecycle \\
Co tourism price in origin country \\
D: dummy variable \\
Dis: travel distance \\
DT: deterministic (linear) trend \\
ER: exchange rate \\
HR: average hotel rate \\
ICR: immigration crime rare \\
INF: capital stock in infrastructure \\
M4: monetary supply \\
ME: marketing expenditure \\
OEI: other economic indicators \\
P: population \\
PB: oil price per barrel \\
PI: price index \\
PREF: the preference index \\
RC: ER adjusted relative price \\
\end{tabular}

RPI: retail price index

SC: substitute price

SF: TC to substitute destinations

SM weighted TM

SPI: Stone's price index

SS: stochastic seasonal component

ST: stochastic trend

TC: travel cost (airfare)

TS: travel cost by surface

TSS: TS to substitute destinations

TTM: total TM

$\mathrm{Y}$ : income in origin country

$\mathrm{Y}_{\mathrm{d}}$ : income in destination country

5 \& 8 Main and alternative models

ADLM: autoregressive distributed lag model

AR(I)MAX autoregressive (integrated)

moving average cause effect model

AR: autoregressive process

BNN: back-prorogation neural network

BSM: non-causal basic structural model

ES: exponential smoothing

FNN: Feed-forward neural network

GSR: Gradual switching regression

LCM: the learning curve model

MA: moving average

NLWSS: nonparametric locally

weighted scatterplot smoothing

SR: static regression

TFM: transfer function model

7. Estimation method

2(3)SLS: two (three)-stage least squares

CORN: Cochbrane-Crcutt procedure

DLS: dynamic least squares

GMM: generalized method of moments

KF Kalman filter algorithm

ML: maximum likelihood

NLLS: non-linear least squares

OLS: ordinary least squares

RIDG: ridge-trace procedure

SUR: seemingly unrelated regression

9. Diagnostic test reported

AC: autocorrelation test

ADF: augmented DF test

ARCH: autoregressive conditional

heteroscedasticity

BC-FF: Box-Cox functional form test

Chow1: structural stability test

Chow2: predictive failure test

$\mathrm{CT}$ : contingency table approach for

directional change error measures

CUSUM: cumulative sum of recursive

residual test for structural stability

CUSUMSQ: cumulative sum of squares

of recursive residuals

DF: Dickey-Fuller unit root test

DW: Durbin-Watson statistic

$\mathrm{E}(l)$ : a statistic to check the goodness of fit

of models in the post-sample period

FU: Forecasting unbiasedness test

HEGY: Hylleberg, Engle, Granger and

Yoo test for seasonal and non-seasonal

unit roots

HESC: heteroscedasticity

HM: Henkiksson-Merton test for

directional change error measures

KPSS: Kwiatkowski-Phillips-Schmidt-

skin unit root test

LD- $\chi^{2}:$ a test for the inclusion of

lagged dependent variables and its

corresponding adjustment path

LM-AC: Lagrange-Multiplier test

MUCL: multicollinearity

Norm: test for non-normality

PEV: prediction error variance

PP: Phillips-Perron unite root test

Q-AC Box-Ljung Q-static

R: normalized correlation coefficient

RESET: mis-specification test

$\mathrm{Z}$ : acceptable output percentage (within

$\pm 15 \%)$ 


\begin{tabular}{|c|c|c|c|c|c|c|c|c|c|}
\hline Study & $\begin{array}{l}\text { Frequency } \\
\& \text { period } 1\end{array}$ & $\begin{array}{l}\text { Region } \\
\text { Focused 2 } \\
\end{array}$ & $\begin{array}{l}\text { Dependent } \\
\text { variable } 3 \\
\end{array}$ & $\begin{array}{l}\text { Independent } \\
\text { Variable } 4\end{array}$ & $\begin{array}{l}\text { Main } \\
\text { Model } 5 \\
\end{array}$ & $\begin{array}{l}\text { Functional } \\
\text { form } 6\end{array}$ & $\begin{array}{l}\text { Estimation } \\
\text { method } 7\end{array}$ & $\begin{array}{l}\text { Alternative } \\
\text { Model } 8 \\
\end{array}$ & $\begin{array}{l}\text { Diagnostic test } \\
\text { Reported } 9\end{array}$ \\
\hline Akal (2004) & $\begin{array}{l}\text { A: } \\
63-01\end{array}$ & Turkey (I) & $\mathrm{TE}$ & TA & AR(I)MAX & $\begin{array}{l}\text { Linear } / \log - \\
\text { linear }\end{array}$ & ML & SR & DW DF Chow1 HESC \\
\hline Akis (1998) & $\begin{array}{l}\text { A: } \\
80-93\end{array}$ & Turkey (I) & TA & Y RC & SR & Log-linear & OLS & & DW \\
\hline $\begin{array}{l}\text { Ashworth \& } \\
\text { Johnson (1990) }\end{array}$ & $\begin{array}{l}\text { A: } \\
72-86\end{array}$ & UK $(\mathrm{O}$ and $\mathrm{D})$ & $\mathrm{TE}(\mathrm{H})$ & Y P RPI D & SR & Log-linear & OLS & & $\begin{array}{l}\text { DW homogeneity } \\
\text { stability }\end{array}$ \\
\hline Bakkal (1991) & $\begin{array}{l}\text { A: } \\
66-85\end{array}$ & Germany $(\mathrm{O})$ & $\mathrm{BS}$ (of TN) & TE/aggregated PI RC SC & $\begin{array}{l}\text { Translog utility } \\
\text { function }\end{array}$ & $\begin{array}{l}\text { Semi-log- } \\
\text { non-linear }\end{array}$ & ML & & Homotheticity additivity \\
\hline Cho (2001) & $\begin{array}{l}\text { Q: } \\
75.1-97.4\end{array}$ & Hong Kong (I) & TA & $\begin{array}{l}\text { ARIMA residuals of GDP } \\
\text { (GNP) CPI IM EX OEIs }\end{array}$ & ARIMAX & & & ARIMA ES & \\
\hline $\begin{array}{l}\text { Crouch et al } \\
\text { (1992) }\end{array}$ & $\begin{array}{l}\text { A: } \\
70-88\end{array}$ & Australia (I) & $\mathrm{TA} / \mathrm{P}$ & Y/P RP TC ME D DT & ADLM & Log-linear & OLS & & DW \\
\hline $\begin{array}{l}\text { De Mello et al } \\
(2002)\end{array}$ & $\begin{array}{l}\text { A: } \\
69-97\end{array}$ & $\begin{array}{l}\text { UK to France, } \\
\text { Portugal Spain }\end{array}$ & BS & TE/P/SPI RC SC D & LAIDS & $\begin{array}{l}\text { Semi-log- } \\
\text { linear }\end{array}$ & SUR & & $\begin{array}{l}\text { DW Homogeneity } \\
\text { symmetry }\end{array}$ \\
\hline Di Matteo (1999) & $\begin{array}{l}\text { Q: } \\
79.1-95.4\end{array}$ & Canada to US & $\mathrm{TE}$ & Y/P ER D & SR & Log-linear & OLS & NLWSS & DW \\
\hline $\begin{array}{l}\text { Di Matteo \& Di } \\
\text { Matteo (1993) }\end{array}$ & $\begin{array}{l}\text { Q: } \\
79.1-89.4\end{array}$ & Canada to US & $\mathrm{TE}$ & Y/P ER RC-gas D & ADLM & Log-linear & OLS & & DW \\
\hline $\begin{array}{l}\text { Divisekera } \\
(2003)\end{array}$ & Not reported & $\begin{array}{l}\text { UK US Japan } \\
\text { New Zealand (O) }\end{array}$ & BS & $\begin{array}{l}\mathrm{TE} / \mathrm{P} / \mathrm{SPI} \text { average of } \mathrm{RC}+\mathrm{TC} \\
\mathrm{SC} \mathrm{D}\end{array}$ & LAIDS & $\begin{array}{l}\text { Semi-log- } \\
\text { linear }\end{array}$ & SUR & & Homogeneity symmetry \\
\hline Dritsakis (2004) & $\begin{array}{l}\text { A: } \\
63-00\end{array}$ & $\begin{array}{l}\text { Germany and UK } \\
\text { to Greece }\end{array}$ & TA & $\mathrm{Y} / \mathrm{P}$ C TC RC & $\mathrm{VAR}(\mathrm{CI}) / \mathrm{ECM}$ & Log-linear & ML OLS & & $\begin{array}{l}\text { ADF DW LM-AC HESC } \\
\text { Chow1,2 RESET Norm }\end{array}$ \\
\hline $\begin{array}{l}\text { Dritsakis \& Atha- } \\
\text { nasiadis }(2000)\end{array}$ & $\begin{array}{l}\text { A: } \\
60-93\end{array}$ & Greece (I) & $\mathrm{TA} / \mathrm{P}$ & $\begin{array}{l}\mathrm{Y} / \mathrm{P} \mathrm{C}_{\mathrm{d}} \mathrm{SC} \text { ER ME DT D } \\
\text { investment }\end{array}$ & ADLM & Log-linear & OLS CORN & & DW \\
\hline $\begin{array}{l}\text { Dritsakis \& Papa- } \\
\text { nastasiou (1998) }\end{array}$ & $\begin{array}{l}\text { A: } \\
60-93\end{array}$ & Greece (I) & TA TE NAC & $\begin{array}{l}\mathrm{C}_{\mathrm{d}} \text { DT TE NAC TA } \\
\text { investment }\end{array}$ & $\begin{array}{l}\text { Simultaneous } \\
\text { equation model }\end{array}$ & Log-linear & 2SLS & & $\begin{array}{l}\text { DW LM-AC RESET } \\
\text { HESC Norm Theil-U }\end{array}$ \\
\hline $\begin{array}{l}\text { Durbarry \& } \\
\text { Sinclair }(2003)\end{array}$ & $\begin{array}{l}\text { A: } \\
68-99\end{array}$ & $\begin{array}{l}\text { France to Italy } \\
\text { Spain UK }\end{array}$ & BS & TE/P/SPI RC SC D & EC-LAIDS & $\begin{array}{l}\text { Semi-log- } \\
\text { linear }\end{array}$ & ML & & $\begin{array}{l}\text { DW Homogeneity } \\
\text { symmetry }\end{array}$ \\
\hline Fujii et al (1985) & $\begin{array}{l}\text { A: } \\
58-80 \\
\end{array}$ & Hawaii (I) & $\begin{array}{l}\text { BS on tourist } \\
\text { goods }\end{array}$ & TE/P/SPI C $\mathrm{C}_{\mathrm{d}}$ DT SC & LAIDS & $\begin{array}{l}\text { Semi-log- } \\
\text { linear }\end{array}$ & ML & & \\
\hline $\begin{array}{l}\text { Gallet \& Braun } \\
(2001)\end{array}$ & $\begin{array}{l}\text { A: } \\
60-85 \\
\end{array}$ & US (O) & TA & $\mathrm{Y} \mathrm{C}_{\mathrm{d}} / \mathrm{ER} \mathrm{SC}$ & GSR & Log-linear & ML CORN & & $\begin{array}{l}\text { DW RESET Durbin } h \\
\text { LD- } \chi^{2}\end{array}$ \\
\hline $\begin{array}{l}\text { Garía-Ferrer \& } \\
\text { Queralt (1997) } \\
\end{array}$ & $\begin{array}{l}\text { M: } \\
79.01-93.12 \\
\end{array}$ & Spain (I) & TE TA & Y RC SC D & STSM & Log-linear & & $\begin{array}{l}\text { ARIMA STSM' } \\
\text { BSM }\end{array}$ & Q-AC \\
\hline $\begin{array}{l}\text { González \& } \\
\text { Moral (1995) }\end{array}$ & $\begin{array}{l}\text { M: } \\
79.01-93.12\end{array}$ & Spain (I) & TE TA & Y RC SC D & STSM & Log-linear & $\mathrm{KF}$ & ARIMA ECM TFM & $\begin{array}{l}\text { r Q-AC HESC Norm } \\
\text { PEV E }(l)\end{array}$ \\
\hline
\end{tabular}




\begin{tabular}{|c|c|c|c|c|c|c|c|c|c|}
\hline $\begin{array}{l}\text { González \& } \\
\text { Moral (1996) }\end{array}$ & $\begin{array}{l}\text { M: } \\
79.01-94.06\end{array}$ & Spain (I) & TE TA & Y RC SC D & STSM & Log-linear & $\mathrm{KF}$ & ARIMA BSM TFM & $\mathrm{r}$ Q-AC HESC Norm $\mathrm{E}(l)$ \\
\hline Greenidge (2001) & $\begin{array}{l}\text { Q: } \\
68.1-97.4 \\
\end{array}$ & Barbados (I) & TA & Y RC SC ST SS Cir & STSM & Log-linear & $\mathrm{KF}$ & BSM & DW Q-AC Norm HESC \\
\hline Holmes (1997) & $\begin{array}{l}\text { M: } \\
81.01-93.08\end{array}$ & $\begin{array}{l}\text { US to British } \\
\text { Columbia }\end{array}$ & TA & Y ER $C_{d} C_{o} D$ & $\begin{array}{l}\text { Intervention \& } \\
\text { TFM }\end{array}$ & Log-linear & OLS & ARIMA & Q-AC \\
\hline Icoz et al (1998) & $\begin{array}{l}\text { A: } \\
82-93 \\
\end{array}$ & Turkey (I) & TA & $\begin{array}{l}\text { Cd ER No. of beds No. of } \\
\text { agents }\end{array}$ & SR & Log-linear & OLS & & DW \\
\hline $\begin{array}{l}\text { Ismail et al } \\
(2000)\end{array}$ & $\begin{array}{l}\text { M: } \\
87.01-97.12 \\
\end{array}$ & Japan to Guam & TA & Y ER TC D & ADLM & Linear & OLS & & \\
\hline Jensen (1998) & $\begin{array}{l}\text { A: } \\
61(70)-95\end{array}$ & Denmark (I) & $\mathrm{TE}$ & Y RC SC D weather & ADLM & Log-linear & OLS & & DW LM-AC DF \\
\hline $\begin{array}{l}\text { Jørgensen \& } \\
\text { Solvoll (1996) } \\
\end{array}$ & $\begin{array}{l}69-93 \\
\text { seasonal }\end{array}$ & Norway (O) & ITC & $\begin{array}{l}\text { Y/P Price of ITC weather } \\
\text { OEI }\end{array}$ & ADLM & Log-linear & OLS CORN & & DW \\
\hline $\begin{array}{l}\text { Kim \& Song } \\
\text { (1998) }\end{array}$ & $\begin{array}{l}\text { A: } \\
62-94\end{array}$ & Korea (I) & TA & Y TC TV RC D & $\mathrm{CI} / \mathrm{ECM}$ & Log-linear & OLS & $\begin{array}{l}\text { Naive } 1 \text { SES MA } \\
\text { AR ARMA VAR }\end{array}$ & $\begin{array}{l}\text { LM-AC CR DW Norm } \\
\text { HESC ARCH RESET } \\
\text { Chow2 Theil-U }\end{array}$ \\
\hline $\begin{array}{l}\text { Kim \& Uysal } \\
(1998)\end{array}$ & $\begin{array}{l}\text { M: } \\
91.01-95.12\end{array}$ & $\begin{array}{l}\text { Hotels in Seoul, } \\
\text { Korea }\end{array}$ & $\mathrm{TN}$ & $C_{d}$ TV No. of events & ARMAX & Log-linear & $\begin{array}{l}\text { 2SLS } \\
\text { CORN }\end{array}$ & & DW Q-AC \\
\hline Kulendran (1996) & $\begin{array}{l}\text { Q: } \\
75.3--95.2 \\
\end{array}$ & Australia (I) & TA & Y RC TC D & $\mathrm{CI} / \mathrm{ECM}$ & Log-linear & ML & & $\begin{array}{l}\text { DW HEGY LM-AC Norm } \\
\text { RESET HESC Chow2 }\end{array}$ \\
\hline $\begin{array}{l}\text { Kulendran \& } \\
\text { King (1997) }\end{array}$ & $\begin{array}{l}\text { Q: } \\
75.1-94.4\end{array}$ & Australia (I) & TA & Y RC TC D & $\mathrm{CI} / \mathrm{ECM}$ & Log-linear & ML & $\begin{array}{l}\text { ARIMA STSM AR } \\
\text { AR(12) SR ARMA }\end{array}$ & DW Theil-U \\
\hline $\begin{array}{l}\text { Kulendran \& } \\
\text { Wilson }(2000)\end{array}$ & $\begin{array}{l}\text { Q: } \\
81.1-97.4\end{array}$ & Australia (I) & TA-B & Y Y $\mathrm{Y}_{\mathrm{d}}$ TA-H TV/Y $\mathrm{Y}_{\mathrm{d}}$ RC IM D & $\mathrm{CI} / \mathrm{ECM}$ & Log-linear & ML OLS & Naive 1 ARIMA & ADF DW LM-AC HEGY \\
\hline $\begin{array}{l}\text { Kulendran \& } \\
\text { Witt (2001) }\end{array}$ & $\begin{array}{l}\text { Q: } \\
78.1-95.3\end{array}$ & UK (O) & $\mathrm{TA} / \mathrm{P}$ & Y/P RC CS TC FS D & $\begin{array}{l}\mathrm{CI} / \mathrm{ECM} \\
\mathrm{ADLM}\end{array}$ & Log-linear & $\begin{array}{l}\text { ML } \\
\text { OLS }\end{array}$ & Naive 1 & $\begin{array}{l}\text { DW LM-AC Chow2 } \\
\text { HESC RESET }\end{array}$ \\
\hline $\begin{array}{l}\text { Kulendran \& } \\
\text { Witt (2003a) }\end{array}$ & $\begin{array}{l}\text { Q: } \\
82.1-98.4\end{array}$ & Australia (I) & TA-B & $\mathrm{Y} \mathrm{Y}_{\mathrm{d}} \mathrm{TA}-\mathrm{H} \mathrm{TV} / \mathrm{Y}_{\mathrm{d}} \mathrm{C}$ & $\begin{array}{l}\text { ECM STSM } \\
\text { BSM }\end{array}$ & Log-linear & ML OLS & $\begin{array}{l}\text { ARIMA }^{1,4} \text { ARIMA }^{1} \\
\text { AR }^{4} \text { Naive }\end{array}$ & ADF DW Q-AC HESC \\
\hline $\begin{array}{l}\text { Kulendran \& } \\
\text { Witt (2003b) }\end{array}$ & $\begin{array}{l}\text { Q: } \\
78.1-95.4\end{array}$ & UK $(\mathrm{O})$ & TA-H & Y RC ER C D & TFM & Log-linear & OLS ML & ECM ARIMA & $\begin{array}{l}\text { DW LM-AC RESET } \\
\text { Chow2 HESC }\end{array}$ \\
\hline $\begin{array}{l}\text { Lanza et al } \\
(2003)\end{array}$ & $\begin{array}{l}\text { A: } \\
75-92 \\
\end{array}$ & $\begin{array}{l}13 \text { European } \\
\text { countries }(\mathrm{O})\end{array}$ & BS & $\begin{array}{l}\text { Total consumption/SPI } \mathrm{C}_{\mathrm{d}} \\
\text { SC D }\end{array}$ & LAIDS & $\begin{array}{l}\text { Semi-log- } \\
\text { linear }\end{array}$ & SUR & & DF PP \\
\hline $\begin{array}{l}\text { Lathiras \& Sirio } \\
\text { poulos (1998) }\end{array}$ & $\begin{array}{l}\text { A: } \\
60-95\end{array}$ & Greece (I) & $\mathrm{TA} / \mathrm{P}$ & Y/P RC SC ER D & $\mathrm{CI} / \mathrm{ECM}$ & Log-linear & ML & & $\begin{array}{l}\text { DW LM-AC RESET } \\
\text { HESC Theil-U CUSUM } \\
\text { CUSUMSQ Chow2 Norm }\end{array}$ \\
\hline Law (2000) & $\begin{array}{l}\text { A: } \\
66-96\end{array}$ & $\begin{array}{l}\text { Taiwan-Hong } \\
\text { Kong }\end{array}$ & TA & Y RC ER P ME HR & $\mathrm{BNN}$ & Non-linear & & $\begin{array}{l}\text { Naive 1 HES MA } \\
\text { FNN SR }\end{array}$ & MAD MSE \\
\hline
\end{tabular}




\begin{tabular}{|c|c|c|c|c|c|c|c|c|c|}
\hline $\begin{array}{l}\text { Law \& Au } \\
(1999)\end{array}$ & $\begin{array}{l}\text { A: } \\
77-97\end{array}$ & $\begin{array}{l}\text { Japan-Hong } \\
\text { Kong }\end{array}$ & TA & RC P ER ME HR & FNN & Linear & & Naive 1 SES MA SR & $\mathrm{Zr}$ \\
\hline $\begin{array}{l}\text { Ledesma-Rodri- } \\
\text { guez et al (2001) }\end{array}$ & $\begin{array}{l}\text { A: } \\
79-97\end{array}$ & Tenerife (I) & TAHA & Y/P ER ME INF PB & ADLM & Log-linear & SUR OLS & SR & DW H-AC HESC \\
\hline Lee et al (1996) & $\begin{array}{l}\text { A: } \\
70-89\end{array}$ & Korea (I) & $\mathrm{TE} / \mathrm{P}$ & Y/P RC ER D & $\mathrm{SR}$ & Log-linear & $\begin{array}{l}\text { OLS CORN } \\
\text { RIDG }\end{array}$ & & DW MUCL \\
\hline Li et al (2002) & $\begin{array}{l}\text { A: } \\
63 / 68-00 \\
\end{array}$ & Thailand (I) & TA & Y RC SCD & $\begin{array}{l}\text { ADLM VAR } \\
\text { CI/ECMs TVP } \\
\end{array}$ & Log-linear & $\begin{array}{l}\text { KF } \\
\text { OLS }\end{array}$ & ARIMA & $\begin{array}{l}\text { ADF LM-AC HESC } \\
\text { Norm Chow1 RESET } \\
\end{array}$ \\
\hline Li et al (2004) & $\begin{array}{l}\text { A: } \\
72-00\end{array}$ & UK (O) & $\mathrm{BS}$ & TE/P/SPI RC SC D & EC-LAIDS & $\begin{array}{l}\text { Semi-log- } \\
\text { linear }\end{array}$ & SUR & Static LAIDS & $\begin{array}{l}\text { DW PP DF Homogeneity } \\
\text { symmetry }\end{array}$ \\
\hline $\begin{array}{l}\text { Lim \& McAleer } \\
(2001)\end{array}$ & $\begin{array}{l}\text { Q: } \\
75.1-96.4\end{array}$ & Australia (I) & TA & Y/P TC ER C RC & VAR CI/VECM & Log-linear & ML OLS & & $\begin{array}{l}\text { ADF HESC LM-AC } \\
\text { Norm Chow1,2 }\end{array}$ \\
\hline $\begin{array}{l}\text { Lim \& McAleer } \\
(2002)\end{array}$ & $\begin{array}{l}\text { A: } \\
75-96\end{array}$ & $\begin{array}{l}\text { Malaysia to } \\
\text { Australia }\end{array}$ & TA & Y/P TC ER C RC & VAR CI/VECM & Log-linear & ML OLS & ADLM & $\begin{array}{l}\text { ADF LM-AC HESC } \\
\text { Norm Chow1,2 }\end{array}$ \\
\hline Lyssiotou (2001) & $\begin{array}{l}\text { Q: } \\
79-91\end{array}$ & UK $(\mathrm{O})$ & BS-H & $\begin{array}{l}\text { TE-H/recreation-PI RC SC } \\
\text { D DT }\end{array}$ & AIDS & $\begin{array}{l}\text { Semi-log- } \\
\text { non-linear }\end{array}$ & NLLS & & $\begin{array}{l}\text { Ac reset homogeneity } \\
\text { symmetry }\end{array}$ \\
\hline $\begin{array}{l}\text { Mangion et al } \\
(2003)\end{array}$ & $\begin{array}{l}\text { A: } \\
73-00\end{array}$ & $\begin{array}{l}\text { UK to Malta } \\
\text { Spain Cyprus }\end{array}$ & $\mathrm{BS}$ & TE/P/SPI RC SC & EC-LAIDS & $\begin{array}{l}\text { Semi-log- } \\
\text { linear }\end{array}$ & SUR & & DW \\
\hline Morley (1996) & $\begin{array}{l}\text { A: } \\
72-92\end{array}$ & Australia (I) & TA-H & $\mathrm{Y} / \mathrm{P} \mathrm{TC}$ & $\mathrm{SR}$ & Linear & $\begin{array}{l}\text { OLS GMM } \\
\text { SUR }\end{array}$ & & DW Norm HESC \\
\hline Morley (1997) & $\begin{array}{l}\text { A: } \\
72-92\end{array}$ & Australia (I) & $\begin{array}{l}\text { TA-H TA- } \\
\text { VFR }\end{array}$ & Y/P RC D TC & ADLM & Log-linear & OLS RELF & & \\
\hline Morley (1998) & $\begin{array}{l}\text { A: } \\
72-92 \\
\end{array}$ & Australia (I) & $\begin{array}{l}\text { TA-H TA- } \\
\text { VFR }\end{array}$ & Y/P RC TC D & ADLM & Non-linear & ML & & \\
\hline $\begin{array}{l}\text { Morris et al } \\
(1995)\end{array}$ & $\begin{array}{l}\text { Q: } \\
81.1-93.4\end{array}$ & Australia (I) & $\begin{array}{l}\text { TA TA-H } \\
\text { TA-VFR }\end{array}$ & $\mathrm{RC} \mathrm{TC} \Delta \mathrm{Y}$ DT D & SR & Log-linear & OLS CORN & & $\begin{array}{l}\text { LM-AC Norm } \\
\text { HESC RESET Chow2 }\end{array}$ \\
\hline $\begin{array}{l}\text { O’Hagan \& } \\
\text { Harrison (1984) }\end{array}$ & $\begin{array}{l}\text { A: } \\
64-81\end{array}$ & US (O) & $\mathrm{BS}$ & TE/P/SPI RC SC D DT & LAIDS & $\begin{array}{l}\text { Semi-log- } \\
\text { linear }\end{array}$ & SUR & & $\begin{array}{l}\text { DW HETE Homogeneity } \\
\text { symmetry }\end{array}$ \\
\hline $\begin{array}{l}\text { Papatheodorou } \\
\text { (1999) }\end{array}$ & $\begin{array}{l}\text { A: } \\
57-90\end{array}$ & $\begin{array}{l}\text { UK, Germany, } \\
\text { France }(\mathrm{O})\end{array}$ & $\mathrm{BS}$ & $\begin{array}{l}\text { TE/tourists/SPI RC SC D } \\
\text { DT }\end{array}$ & LAIDS & $\begin{array}{l}\text { Semi-log- } \\
\text { linear }\end{array}$ & SUR & & DW \\
\hline $\begin{array}{l}\text { Payne \& Mervar } \\
(2002)\end{array}$ & $\begin{array}{l}\text { Q: } \\
\text { 93.1-99.4 }\end{array}$ & Croatia (I) & $\mathrm{TE}$ & Y RC D & SR & Log-linear & OLS & & $\begin{array}{l}\text { DW LM-AC Norm } \\
\text { HESC RESET CUSUM } \\
\text { CUSUMSQ }\end{array}$ \\
\hline Pyo et al (1991) & $\begin{array}{l}\text { A: } \\
72-87\end{array}$ & US domestic & $\begin{array}{l}\text { TE on tourist } \\
\text { goods }\end{array}$ & $\mathrm{TE} / \mathrm{SPI} \mathrm{C}_{\mathrm{d}} \mathrm{SC}$ & LES & $\begin{array}{l}\text { Semi-log- } \\
\text { linear }\end{array}$ & SUR OLS & & \\
\hline $\begin{array}{l}\text { Qiu \& Zhang } \\
\text { (1995) }\end{array}$ & $\begin{array}{l}\text { A: } \\
75-90\end{array}$ & Canada (I) & TA TE & Y/P ER $C_{d}$ D DT ICR & SR & Linear & OLS & log-linear SR & BC-FF \\
\hline $\begin{array}{l}\text { Qu \& Lam } \\
\text { (1997) }\end{array}$ & $\begin{array}{l}\text { A: } \\
84-95\end{array}$ & $\begin{array}{l}\text { Mainland China } \\
\text { to Hong Kong }\end{array}$ & TA & Y/P C ER D & SR & Linear & OLS & & DW MUCL \\
\hline
\end{tabular}




\begin{tabular}{|c|c|c|c|c|c|c|c|c|c|}
\hline $\begin{array}{l}\text { Riddington } \\
\text { (1999) }\end{array}$ & $\begin{array}{l}\text { A: } \\
73-94\end{array}$ & UK Skiing place & TA & M4 DT & TVP & Linear & $\mathrm{KF}$ & $\begin{array}{l}\text { LCM Static } \\
\text { regression }\end{array}$ & DW \\
\hline $\begin{array}{l}\text { Rosselló-Nadal } \\
(2001)\end{array}$ & $\begin{array}{l}\text { M: } \\
75.01-99.12\end{array}$ & $\begin{array}{l}\text { Balearic Islands } \\
\text { (I) }\end{array}$ & TA & ER RC OEIs & $\begin{array}{l}\text { ADLM(Leading } \\
\text { indicator) }\end{array}$ & Linear & OLS & Naive1 ARIMA & $\begin{array}{l}\text { DW Q-AC LM-AC AIC } \\
\text { SC }\end{array}$ \\
\hline $\begin{array}{l}\text { Rosselló-Nadal et } \\
\text { al (2004) }\end{array}$ & $\begin{array}{l}\text { M/A: } \\
82-01\end{array}$ & $\begin{array}{l}\text { The Balearic } \\
\text { Islands (I) }\end{array}$ & $\begin{array}{l}\text { Gini- } \\
\text { coefficient }\end{array}$ & Y/P ER RC & ECM & Linear & DLS & & DW LM-AC AIC SC \\
\hline $\begin{array}{l}\text { Shan \& Wilson } \\
(2001)\end{array}$ & $\begin{array}{l}\text { M: } \\
87.01-98.01\end{array}$ & China (I) & $\begin{array}{l}\text { TA Y RC } \\
\text { IM+EX ER }\end{array}$ & TA Y RC IM+EX ER & VAR & Linear & SUR & & $\begin{array}{l}\text { ADF Modified Wald AIC } \\
\text { SC }\end{array}$ \\
\hline Sheldon (1993) & $\begin{array}{l}\text { A: } \\
70-86 \\
\end{array}$ & US (I) & $\mathrm{TE}$ & Y ER RC SC & SR & $\begin{array}{l}\text { Linear } / \log \text { - } \\
\text { linear }\end{array}$ & OLS & $\begin{array}{l}\text { Naïve1,2 Trend } \\
\text { fitting models }\end{array}$ & \\
\hline $\begin{array}{l}\text { Smeral et al } \\
(1992)\end{array}$ & $\begin{array}{l}\text { A: } \\
75-00\end{array}$ & $\begin{array}{l}18 \text { OECD } \\
\text { countries }(\mathrm{I} / \mathrm{O})\end{array}$ & $\begin{array}{l}\text { TM } \\
\text { TX }\end{array}$ & $\begin{array}{l}Y_{\text {Y C }} \text { D } \\
\text { TTX RC D (ST) }\end{array}$ & $\begin{array}{l}\text { complete } \\
\text { system }\end{array}$ & linear & & & DW \\
\hline $\begin{array}{l}\text { Smeral \& Weber } \\
(2000)\end{array}$ & $\begin{array}{l}\text { A: } \\
75-96 \\
\end{array}$ & $\begin{array}{l}20 \text { OECD } \\
\text { countries }(\mathrm{I} / \mathrm{O})\end{array}$ & $\begin{array}{l}\text { TM } \\
\text { TX }\end{array}$ & $\begin{array}{l}\text { Y RC D (DT) } \\
\text { SM RC D (DT) }\end{array}$ & $\begin{array}{l}\text { complete } \\
\text { system }\end{array}$ & linear & OLS & & DW \\
\hline $\begin{array}{l}\text { Smeral \& Witt } \\
(1996)\end{array}$ & $\begin{array}{l}\text { A: } \\
75-94\end{array}$ & $\begin{array}{l}18 \text { OECD } \\
\text { countries }(\mathrm{I} / \mathrm{O})\end{array}$ & $\begin{array}{l}\text { TM } \\
\text { TX }\end{array}$ & $\begin{array}{l}\mathrm{Y} \mathrm{C}_{\mathrm{o}} \mathrm{D} \\
\text { SM RC D }\end{array}$ & $\begin{array}{l}\text { complete } \\
\text { system }\end{array}$ & Linear & $\begin{array}{l}\text { OLS } \\
\text { Sauss-Seidel } \\
\end{array}$ & & DW \\
\hline Song et al (2000) & $\begin{array}{l}\text { A: } \\
65-94\end{array}$ & UK (O) & TA/P-H & Y/P RC SC PREF D & $\mathrm{CI} / \mathrm{ECM}$ & Log-linear & OLS & $\begin{array}{l}\text { Naive } 1 \text { MA AR } \\
\text { ARMA VAR }\end{array}$ & $\begin{array}{l}\text { ADF PP Norm ARCH } \\
\text { DW LM-AC RESET } \\
\text { HESC Chow2 }\end{array}$ \\
\hline $\begin{array}{l}\text { Song, Witt \& Li } \\
(2003)\end{array}$ & $\begin{array}{l}\text { A: } \\
63 / 68-00 \\
\end{array}$ & Thailand (I) & TA & Y RC SC D & $\begin{array}{l}\text { ADLM VAR } \\
\text { CI/ECMs TVP }\end{array}$ & Log-linear & $\begin{array}{l}\text { KF } \\
\text { OLS }\end{array}$ & ARIMA Naive 1 & $\begin{array}{l}\text { ADF LM-AC HESC } \\
\text { Norm Chow1 RESET }\end{array}$ \\
\hline $\begin{array}{l}\text { Song, Witt \& } \\
\text { Jensen (2003) }\end{array}$ & $\begin{array}{l}\text { A: } \\
69-97\end{array}$ & Denmark (I) & $\mathrm{TA} / \mathrm{P}-\mathrm{H}$ & TE/P RC SC TC DT D & $\mathrm{CI} / \mathrm{ECM}$ & Log-linear & OLS & $\begin{array}{l}\text { SR Naive } 1 \text { ARIMA } \\
\text { VAR ADLM TVP }\end{array}$ & $\begin{array}{l}\text { LM-AC Norm HESC } \\
\text { RESET Chow1 }\end{array}$ \\
\hline $\begin{array}{l}\text { Song \& Witt } \\
(2003)\end{array}$ & $\begin{array}{l}\text { A: } \\
62-98\end{array}$ & Korea (I) & TA & Y TV RC SC D & ADLM ECM & Log-linear & OLS & & $\begin{array}{l}\text { LM-AC Norm HESC } \\
\text { RESET }\end{array}$ \\
\hline $\begin{array}{l}\text { Song \& Wong } \\
\text { (2003) }\end{array}$ & $\begin{array}{l}\text { A: } \\
73-00\end{array}$ & Hong Kong (I) & TA & Y RC SC & TVP & Log-linear & KF & & AIC SC LL \\
\hline $\begin{array}{l}\text { Song, Wong \& } \\
\text { Chon (2003) }\end{array}$ & $\begin{array}{l}\text { A: } \\
73(81)-00\end{array}$ & Hong Kong (I) & TA & Y RC SC D & ADLM & Log-linear & OLS & & $\begin{array}{l}\text { LM-AC Norm } \\
\text { HESC RESET Chow2 }\end{array}$ \\
\hline $\begin{array}{l}\text { Syriopoulos } \\
(1995)\end{array}$ & $\begin{array}{l}\text { A: } \\
62-87 \\
\end{array}$ & $\begin{array}{l}\text { Mediterranean } \\
\text { countries (I) } \\
\end{array}$ & $\mathrm{TE}$ & Y/P C RC ER D DT(ST) & $\mathrm{CI} / \mathrm{ECM}$ & Log-linear & OLS & & $\begin{array}{l}\text { LM-AC ARCH HESC } \\
\text { Chow1 }\end{array}$ \\
\hline $\begin{array}{l}\text { Syriopoulos \& } \\
\text { Sinclair (1993) }\end{array}$ & $\begin{array}{l}\text { A: } \\
60-87 \\
\end{array}$ & $\begin{array}{l}\text { US UK Germany } \\
\text { France Sweden }(\mathrm{O})\end{array}$ & BS & TE/P/SPI RC SC D DT & LAIDS & $\begin{array}{l}\text { Semi-log- } \\
\text { linear }\end{array}$ & OLS SUR & & $\begin{array}{l}\text { DW Homogeneity } \\
\text { symmetry }\end{array}$ \\
\hline $\begin{array}{l}\text { Turner et al } \\
(1998)\end{array}$ & $\begin{array}{l}\text { Q: } \\
78.1-95.4\end{array}$ & UK (O) & $\begin{array}{l}\text { TA-B TA-H } \\
\text { TA-VFR }\end{array}$ & $\begin{array}{l}\text { Y/C RC SC TC SF IM EX } \\
\text { OEIs }\end{array}$ & $\begin{array}{l}\text { Structural } \\
\text { equation model }\end{array}$ & Linear & & & MUCL \\
\hline $\begin{array}{l}\text { Turner \& Witt } \\
\text { (2001a) }\end{array}$ & $\begin{array}{l}\text { Q: } \\
78.1-97.4\end{array}$ & New Zealand (I) & $\begin{array}{l}\text { TA-B TA-H } \\
\text { TA-VFR }\end{array}$ & $\begin{array}{l}\text { Y P RC TC SFTV/Y } \mathrm{I}_{\mathrm{IM}} \\
\text { EX, OEIs }\end{array}$ & $\begin{array}{l}\text { Structural } \\
\text { equation model }\end{array}$ & Linear & & & MUC \\
\hline $\begin{array}{l}\text { Turner \& Witt } \\
(2001 b)\end{array}$ & $\begin{array}{l}\text { Q: } \\
78.2-98.3\end{array}$ & New Zealand (I) & $\begin{array}{l}\text { TA TA-B TA- } \\
\text { HTA-VFR }\end{array}$ & $\mathrm{Y} \mathrm{RC} \mathrm{TC} \mathrm{TV} / \mathrm{Y}_{\mathrm{d}}$ & STSM & Log-linear & KF & BSM & Norm HESC DW Q-AC $r$ \\
\hline
\end{tabular}




\begin{tabular}{|c|c|c|c|c|c|c|c|c|c|}
\hline $\begin{array}{l}\text { Uysal \& Roubi } \\
(1999)\end{array}$ & $\begin{array}{l}\text { Q : } \\
81.1-96.4\end{array}$ & Canada to US & $\mathrm{TE}$ & Y/P RC D & ADLM & Log-linear & OLS & FNN & \\
\hline $\begin{array}{l}\text { Vanegas \& Croes } \\
(2000)\end{array}$ & $\begin{array}{l}\text { A: } \\
75-96\end{array}$ & US to Aruba & TA & Y RC ER & ADLM & Log-linear & OLS & Linear & DW predictive efficiency \\
\hline $\begin{array}{l}\text { Var \& Icoz } \\
(1990)\end{array}$ & $\begin{array}{l}\text { A: } \\
79-87\end{array}$ & Turkey (I) & TA & Y/P ER Dis & SR & Log-linear & OLS & & \\
\hline $\begin{array}{l}\text { Vogt \& Wittaya- } \\
\text { korn (1998) }\end{array}$ & $\begin{array}{l}\text { A: } \\
60-93\end{array}$ & Thailand (I) & $\mathrm{TE}$ & RC ER Y & $\mathrm{CI} / \mathrm{ECM}$ & Log-linear & OLS & & $\mathrm{ADF}$ \\
\hline Webber (2001) & $\begin{array}{l}\text { Q: } \\
83.1-97.4\end{array}$ & Australia (O) & TA & $\begin{array}{l}\mathrm{Y} \mathrm{RC} \mathrm{C}_{\mathrm{d}} / \mathrm{ER} \text { (C ER) ER- } \\
\text { volatility }\end{array}$ & $\mathrm{CI} / \mathrm{VAR}$ & Log-linear & OLS & & ADF PP symmetry \\
\hline White(1985) & $\begin{array}{l}\text { A: } \\
54-81\end{array}$ & US (O) & BS & TE/P/SPI RC TC SC D DT & LAIDS & $\begin{array}{l}\text { Semi-log- } \\
\text { linear }\end{array}$ & ML & & Homogeneity symmetry \\
\hline Witt et al (2003) & $\begin{array}{l}\text { A: } \\
69-97\end{array}$ & Denmark (I) & TA/P-H & TE/P RC SC TC DT D & $\mathrm{CI} / \mathrm{ECM}$ & Log-linear & OLS & $\begin{array}{l}\text { Naive } 1 \text { ARIMA SR } \\
\text { VAR ADLM TVP }\end{array}$ & FU HM CT \\
\hline $\begin{array}{l}\text { Witt \& Witt } \\
(1990)\end{array}$ & $\begin{array}{l}\text { A: } \\
65-80\end{array}$ & $\begin{array}{l}\text { European } \\
\text { countries, US (I/O) }\end{array}$ & $\mathrm{TA} / \mathrm{P}$ & $\begin{array}{l}\text { Y/P C } C_{d} \text { SC ER TC SF TS } \\
\text { TSS D DT }\end{array}$ & SR & Log-linear & OLS CORN & & DW MSPE \\
\hline $\begin{array}{l}\text { Witt \& Witt } \\
\text { (1991) }\end{array}$ & $\begin{array}{l}\text { A: } \\
65-85\end{array}$ & $\begin{array}{l}\text { France, Germany, } \\
\text { UK, US (O) }\end{array}$ & TA/P TA & $\begin{array}{l}\text { Y/P C SC ER, TC SF TS } \\
\text { TSS D }\end{array}$ & SR & Log-linear & OLS & Time series models & \\
\hline $\begin{array}{l}\text { Witt \& Witt } \\
(1992)\end{array}$ & $\begin{array}{l}\text { A: } \\
65-80\end{array}$ & $\begin{array}{l}\text { France Germany } \\
\text { UK US }(\mathrm{O})\end{array}$ & $\mathrm{TA} / \mathrm{P}$ & Y/P C $\mathrm{d}_{\mathrm{d}}$ CS ER TC SF D & ADLM & Log-linear & OLS CORN & $\begin{array}{l}\text { Naive } 1,2 \text { AR ES } \\
\text { ARIMA Trends }\end{array}$ & DW \\
\hline
\end{tabular}




\section{Measures of Tourism Demand}

Compared to tourism demand studies prior to 1990, the measures of tourism demand have not changed much. Tourist arrivals was still the most common measure in the last decade, followed by the tourist expenditure. In particular, tourist expenditure, in the form of either absolute values or budget shares, is required by the specification of demand system models, such as the linear expenditure system (LES) and the AIDS.

Compared with the tourism literature before 1990, recent studies pay more attention to disaggregated tourism markets by travel purpose (for example, Morley 1998; Turner et al 1998; Turner and Witt 2001a). Amongst various market segments, leisure tourism attracted the most research attention. 12 studies focused on this particular tourism market (for example, Ashworth and Johnson 1990; Kulendran and Witt 2003b; Song, Romilly, and Liu 2000; Song, Witt, and Li 2003). Different market segments are associated with different influencing factors and varying decision-making processes. Therefore, studies at disaggregated levels give more precise insights into the features of the particular market segments. As a result, more specific and accurate information can be provided to develop efficient marketing strategies.

\section{Explanatory Variables}

Consistent with previous tourism demand studies, income, relative prices, substitute prices, travel costs, exchange rates, dummies and deterministic trends were the most frequently considered influencing factors in the reviewed studies. In spite of different definitions of income and relative prices, both of them were shown to be the most significant determinants for international tourism demand. Although travel costs had been considered in over $50 \%$ of the studies reviewed by both Crouch and Lim, in recent studies they did not attract as much attention as before, with only 24 studies including this variable. As precise measurements of travel costs were lacking, especially of the aggregate level, proxies such as airfares between the origin and the destination had to be used. However, only in a few cases did the use of proxies result in significant coefficient estimates. Another reason for insignificant effects of travel costs may be related to all inclusive tours where charter flights are often used, and hence airfares bear little relation to published scheduled fares. The deterministic trend variable describes a steady change format, which is too restrictive to be realistic and may cause serious multicollinearity problems. With this borne in mind, recent studies have been less keen to include it in model specifications. This variable only appeared in 11 reviewed studies. To capture the effects of one-off events, dummy variables have been commonly used. The two oil crises in the 1970s were shown to have the most significant adverse impacts on international tourism demand, followed by the Gulf War in the early 1990s, and the global economic recession in the mid 1980s. Other regional events and origin/destination-specific affairs have also been taken into account in specific studies.

It should be noted that no effort has been made to examine the impact of tourism supply in the tourism demand literature, which means that the problem of identification has been ignored. An implicit assumption of this omission is that the tourism sector concerned is assumed to be sufficiently small and the supply elasticity is infinite. To draw more robust conclusions with regard to demand elasticity analysis, however, this condition needs to be carefully examined in future studies. 


\section{Functional Forms}

Continuing the trend of the 1960s-1980s, log-linear regression was still the predominant functional form in the context of tourism demand studies in the 1990s. 53 studies specified log-linear models, 17 linear models, and only 3 non-linear forms. In addition, a semi-log (both linear and non-linear) form appeared in 14 demand systems, principally AIDS models, where only independent variables (prices and real expenditure) were transformed into logarithm. Crouch (1992) concluded that the loglinear form was generally proved to be superior when both linear and log-linear forms were tested. In a recent study, Vanegas and Croes (2000) compared a few linear and log-linear models of US demand for tourism in Aruba and concluded that log-linear models generally fitted the data better (although only slightly) in terms of the statistical significance of the estimated coefficients, whereas Qiu and Zhang (1995) ran a similar comparison but did not find a significant difference between the two forms.

An advantage of using log-linear regressions is that the log transformation may reduce the integration order of the variables from $\mathrm{I}(2)$ to $\mathrm{I}(1)$, so that the standard cointegration (CI) analysis is allowed. However, the elasticities derived from loglinear regressions (within the traditional fixed-parameter framework) are constant over time. This condition is quite restrictive and often leads to the failure of dynamic analysis of tourism demand. Moreover, such a model may not be useful for short-term forecasting (Lim 1997b). However, the problem of constant elasticities can be solved by rewriting the regression in the state space form (SSF) and estimating it by the Kalman filter algorithm. Such a method is termed the TVP model, and it will be introduced in the following section.

\section{Model Specification and Estimation}

CI Model and Error Correction Model (ECM). In the early 1990s, econometric modeling and forecasting of tourism demand was still restricted to static models, which suffer from quite a few problems such as spurious regression (Song and Witt, 2000). Since the mid 1990s, dynamic models, for example, a number of specific forms of the autoregressive distributed lag models (ADLMs), (Hendry 1995, p. 232) including ECMs have appeared in the tourism demand literature. The potentially spurious regression problem can be readily overcome by using the $\mathrm{CI} / \mathrm{ECM}$ analysis. When the CI relationship is identified, the CI equation can be transformed into an ECM (and vice versa), in which both the long-run equilibrium relationship and shortrun dynamics are traced. An additional advantage of using the ECM is that the regressors in an ECM are almost orthogonal and this avoids the occurrence of multicollinearity, which may otherwise be a serious problem in econometric analysis (Syriopoulos, 1995). However, it should be noted the CI relationship does not necessarily hold in every case of tourism demand. The application of this methodology should be subject to strict statistical tests.

17 of the studies under review applied the CI/ECM technique to international tourism demand analysis. Four CI/ECM estimation methods have been used - the Engle-Granger (1987) two-stage approach (EG), the Wickens-Breusch (1988) onestage approach (WB), the ADLM approach (Pesaran and Shin, 1995), and the most frequently employed Johansen (1988) maximum likelihood (JML) approach. Due to 
different modeling strategies, these models may yield demand elasticities with large discrepancies for the same data set. Moreover, unlike the other methods, the JML approach may detect more than one CI relationship amongst the demand and explanatory variables. The determination of the unique CI relationships in the JML framework involves testing for identification. It is important to impose appropriate identifying restrictions, which should have an explicit underpinning of economic theories (Harris and Sollis 2003). All of these approaches have their merits, and there has not been clear-cut evidence to show that any one is superior to the others. Sometimes the evaluation is associated with their ex post forecasting performance.

Time Varying Parameter (TVP) Model. To overcome the unrealistic assumption of constant coefficients (or elasticities in log-linear regression) associated with the traditional econometric techniques, the TVP model was developed and has been applied in tourism demand studies. The TVP model is specified in the following SSF:

$$
\begin{gathered}
y_{t}=x_{t} \alpha_{t}+\varepsilon_{t} \\
\alpha_{t+1}=T_{t} \alpha_{t}+R_{t} \eta_{t}
\end{gathered}
$$

where $y_{t}$ is a vector of tourism demand, $x_{t}$ is an matrix of explanatory variables, $\alpha_{t}$ is an unobserved vector of parameters known as the state vector, $T_{t}$ and $R_{t}$ are transition matrices, and $\varepsilon_{t}$ and $\eta_{t}$ are vectors of Gaussian disturbances which are serially independent and independent of each other at all time points. The TVP model can be estimated using the Kalman filter algorithm. The TVP model first appeared in the tourism literature in 1999 and only 6 applications have been identified. They were all related to annual tourism data and the main focus of these studies was the evolution of demand elasticities over a relatively long period. Taking sufficient account of the dynamics of tourist behaviors, the TVP model is likely to generate more accurate forecasts of tourism demand. This will be discussed in a later section.

Vector Autoregressive (VAR) Approach. Most of the traditional tourism demand models are specified in a single-equation form, which implicitly assumes that the explanatory variables are exogenous. If the assumption is invalid, the estimated parameters are likely to be biased and inconsistent. Where exogeneity is not assured, the vector autoregressive (VAR) model is more appropriate. The VAR model is a system of equations in which all variables are treated as endogenous. It can be written as:

$$
Y_{t}=\sum_{i=1}^{p} A_{i} Y_{t-i}+B Z_{t}+U_{t}
$$

where $Y_{t}$ is a $k$ vector of endogenous variables, $Z_{t}$ is a $d$ vector of exogenous variables, $A_{i}$ and $B$ are matrices of coefficients to be estimated, and $U_{t}$ is a vector of innovations that is independently and identically distributed. The JML CI/ECM analysis is based on the unrestricted VAR method. Since 1998, there have been 8 studies utilizing the VAR approach including the cointegrated VAR and VECM for tourism demand analysis.

Almost Ideal Demand Systems (AIDS). Another limitation of the single-equation analysis of tourism demand is that this approach is incapable of analyzing the interdependence of budget allocations to different tourist products/destinations. 
Lacking a strong underpinning of economic theory, the single-equation approach is relatively ad hoc. As a result, it is hard to attach a strong degree of confidence to the results (especially regarding demand elasticities) derived from this methodology. On the contrary, the demand system approach, which embodies the principles of demand theory, is more appropriate for tourism demand analysis. Amongst a number of system approaches available, the AIDS introduced by Deaton and Muellbauer (1980) has been the most commonly used method because of its considerable advantages over others. The AIDS model is specified in the form:

$$
w_{i}=\alpha_{i}+\sum_{j} \gamma_{i j} \log p_{j}+b_{i} \log (x / P)+u_{i}
$$

where $\mathrm{w}_{i}$ is the budget share of the $i$ th good, $p_{i}$ is the price of the $i$ th good, $x$ is total expenditure on all goods in the system, $P$ is the aggregate price index for the system, and $u_{i}$ is the disturbance term. The aggregate price index $P$ is defined as:

$$
\log P=a_{0}+\sum_{i} \alpha_{i} \log p_{i}+\frac{1}{2} \sum_{i} \sum_{j} \gamma_{i j} \log p_{i} \log p_{j}
$$

where $a_{0}$ and $\alpha_{i}$ are parameters that to be estimated. Replacing $P$ with the following Stone's (1954) price index $\left(P^{*}\right)$, the linearly approximated AIDS is derived and termed "LAIDS".

$$
\log P^{*}=\sum_{i} w_{i} \log p_{i}
$$

The AIDS/LAIDS can be used to test the properties of homogeneity and symmetry associated with demand theory. Moreover, both uncompensated and compensated demand elasticities including expenditure, own-price and cross-price elasticities can be calculated. They have a stronger theoretical basis than the single-equation approach.

The LAIDS model can be estimated by three methods: ordinary least squares (OLS), maximum likelihood (ML) and Zellner's (1962) iterative approach for seemingly unrelated regression (SUR) estimation. The SUR method is used most often, as it performs more efficiently than OLS in the system with the symmetry restriction (Syriopoulos 1995). It will also converge to the ML estimator, provided that the residuals are distributed normally (Rickertsen 1998).

Since the AIDS model was introduced into tourism demand studies in the 1980s, it has not attracted much attention until recently. 12 applications have been identified including 3 in the 1980s, 1 in 1993 and 8 after 1999. Most of these studies analyzed allocations of tourists' expenditure in a group of destination countries, while Fujii et al (1985) investigated tourists' expenditure on different consumer goods in a particular destination. Where a group of destinations are concerned, substitutability and complementarity between them are investigated by calculating cross-price elasticities. The AIDS/LAIDS has been developed from the original static form to the error correction form. Combing the ECM with the LAIDS, Durbarry and Sinclair (2003), Li, Song, and Witt (2004), and Mangion, Durbarry, and Sinclair (2003) specified EC-LAIDS models to examine the dynamics of tourists' consumption behavior. 
Other demand system models such as the LES by Pyo, Uysal, and McLellan (1991) and the translog utility function by Bakkal (1991) have also appeared in the tourism context, but compared to AIDS/LAIDS their applications were extremely rare.

Time Series Models Augmented with Explanatory Variables. Another emerging trend of tourism demand research has been the introduction of the advanced timeseries techniques into the causal regression framework. By doing so, the advantages of both methodologies are combined. Two notable examples are the structural time series model with explanatory variables (STSM) which expands the basic structural model without explanatory variables (BSM), and the AR(I)MAX model based on the AR(I)MA technique. The BSM and the AR(I)MA model are advanced time-series forecasting techniques and have shown favorable forecasting performance in the tourism context. The BSM can readily capture the trends, seasonal patterns and cycles involved in demand variables. Similar to the technique of the TVP model, the BSM and STSM are also written in the SSF and estimated by the Kalman filter. They are very useful as far as seasonal data are concerned. The AR(I)MA model includes both autoregressive filters and moving average filters to account for systematic effects and shock effects in the endogenous variable itself, respectively. With explanatory variables being added into the model specifications, the STSM and the AR(I)MAX model are more powerful in interpreting variations in demand variables relative to the BSM and the AR(I)MA model, respectively. Meanwhile, they embody the dynamics of the demand variables and overcome the problem of autocorrelation suffered by conventional static regressions. Amongst the 84 econometric studies, there are 6 applications of the STSM and 3 of the AR(I)MAX model. Another advantage of using these models is the potential to generate accurate tourism forecasts, which will be investigated in a later section.

Data frequency affects the specification of the models. For example, the STSM and AR(I)MAX models have been used more often when monthly or quarterly data are concerned. Annual data, however, have always been used in the estimation of the AIDS/LAIDS models. Annual data, however, have always been used in the estimation of AIDS/LAIDS models. The main reason for this is that these latter models aim to examine long-run demand elasticities. In most cases, the TVP model has been applied to annual data, although it is possible to incorporate seasonality into the specification. The combination of the TVP model with the STSM is of interest for future tourism demand studies. Depending on the integration order of the data, the ECM and VAR models can readily accommodate data with different frequencies (Song and Witt 2000).

\section{Diagnostic Tests}

Witt and Witt (1995) pointed out the problems in tourism demand models prior to the early 1990s, one of which is the lack of diagnostic checking. As a result, the inferences from the estimated models might be highly sensitive to the statistical assumptions, especially when a small number of observations are available (Lim 1997a). The situation has changed since the mid 1990s. In addition to the conventional statistics reported in earlier studies such as goodness of fit, $F$ statistic and Durbin-Watson autocorrelation statistic, many recent studies have carried out tests for unit roots, higher-order autocorrelation, heteroscedasticity, non-normality, mis-specification, structural break and forecasting failure. In particular, Dristakis (2003), Kim and Song (1998), and Song, Romilly, and Liu (2000) each reported about 
10 diagnostic tests for their estimates. Amongst various diagnostic tests, unit root tests for annual data or seasonal unit root tests for monthly or quarterly data have been widely used where CI/ECM approaches were considered. Most of the models reported in the studies after 1995 passed the majority of these tests. The enhanced model performance is likely to generate more accurate forecasts and more meaningful implications for the practical operations of tourism industries and government agencies.

\section{Demand Elasticities}

Tourism demand elasticities have been discussed comprehensively by Crouch (1992, 1994a, 994b, 1995, 1996). Consistent with his findings, recent studies have also shown that the income elasticity is generally greater than one, indicating that international tourism, especially long-haul travel, is a luxury. The own-price elasticity is normally negative, although the magnitudes vary considerably. The reasons that cause the discrepancies in demand elasticities have been identified in Crouch's work, therefore this paper will only address some additional issues.

Long-Run and Short-Run Elasticities. In addition to the findings in line with previous studies, some new light has been shed on the literature by the research adopting the $\mathrm{CI} / \mathrm{ECM}$ techniques. Given the $\mathrm{CI}$ relationship being assured by statistical tests, long-run and short-run tourism demand elasticities can be calculated from the $\mathrm{CI}$ equation and the ECM, respectively. With regard to the income elasticity, lower degrees of significance in ECMs than those in the CI models indicate that income affects tourism demand more in the long run than in the short run. To some extent, it indicates that Friedman's (1957) permanent income hypothesis holds. In other words, consumption depends on what people expect to earn over a considerable period of time, and fluctuations in income regarded as temporary have little effect on their consumption spending. Many empirical studies also show that the values of both the income and own-price elasticities in the long run are greater than their short-run counterparts, suggesting that tourists are more sensitive to income/price changes in the long run than in the short run. These findings are in line with demand theory. Due to information asymmetry and relatively inflexible budget allocations, it takes time before income changes affect tourism demand (Syriopoulos 1995).

Cross-Price Elasticities. The cross-price elasticity contributes to the analysis of the interrelationships between alternative destinations. As mentioned earlier, this is one of the advantages of the AIDS model over single-equation regressions. Seven studies used this approach to study UK outbound tourism demand. Table 2 summarizes the substitution and complementarity relationships between alternative destinations considered by UK tourists. Due to the differences with respect to the composition of the demand systems, the data periods, the definitions of variables and estimation methods, some contradictions between the findings are identified. However, some findings are supported across studies. For example, a significant substitution effect between France and Spain was commonly found (see De Mello, Park and Sinclair 2002, Li, Song, and Witt 2004, Lyssiotou 2001), and Greece and Italy were generally regarded as complementary destinations by UK tourists (see Li, Song, and Witt 2004, Lyssiotou 2001, Papatheodorou 1999, Syriopoulos and Sinclair 1993). Moreover, Italy and Turkey were substitutes for each other to some extent (see Papatheodorou 1999, Syriopoulos and Sinclair 1993). These findings have important policy implications for the destination concerned. A significant substitution effect indicates 
strong competitors, and different degrees of substitution (suggested by the values of the elasticities $\varepsilon_{a b}$ and $\varepsilon_{b a}$ ) between the competing destinations $a$ and $b$ show their competitive positions in the tourism markets. Therefore, the implication could be to adopt appropriate strategies based on the specific attributes the destinations possess or to focus on differentiated markets segments, i.e., to make full use of their competitive advantages. Where complementary effects are in place, the destinations involved may consider launching joint marketing programs to maximize their total profits.

Evolution of Eelasticities. Compared to the long-run constant demand elasticities, analyzing the evolution of demand elasticities over time has great importance for short-term forecasting. Crouch $(1994 b, 1996)$ has identified the differences regarding income and own-price elasticities in different time periods. Using the TVP approach, Li, Song, and Witt (2002), Song and Witt (2000), and Song and Wong (2003) confirmed the above findings in their empirical studies. In particular, the significant impacts of the two oil crises in the 1970s and the economic recession in the 1980s on tourism demand, in terms of the income elasticities, were readily accommodated in their models. It suggests that the TVP model is preferable to the log-linear fixedparameter regressions when investigating the dynamics of tourism demand.

\section{PERFORMANCE OF FORECASTING MODELS}

Among the 84 studies being reviewed, 23 papers exercised the compared forecasting performance amongst different econometric models or amongst econometric, univariate time-series and other (e.g. neural network) models. Apart from Rossello-Nadal (2001) who investigated forecasting models' turning point accuracy, all the other papers examined forecast error magnitudes. Therefore, the review of forecasting models' performance will focus on error magnitude accuracy. In addition to error magnitudes, Witt, Song, and Louvieris (2003) also observed directional changes of demand forecasts, and Witt and Witt (1991) and its extended version Witt and Witt (1992) included directional changes and trend changes in their forecasting accuracy evaluations. Due to extremely small numbers of applications, these two measures of forecasting accuracy are ignored in this review. The ranks of compared models in each of the 22 studies ${ }^{1}$, in terms of forecast accuracy, are tabulated for detailed analysis (Table 3). Since error magnitude accuracy dominates the evaluation of tourism demand forecasting, the following discussion will mainly focus on this measure.

Table 3 summarizes the rankings of forecasting models measured by the MAPE except for 4 studies in which only the MAE or RMSE was available. Due to space limitations, the results of other measures are omitted from this table. The rankings of competing models at each forecasting horizon and the overall ranks are presented in Table 3. Where they were not reported directly in the original papers, the aggregation of MAPEs is calculated based on the individual MAPEs originally reported.

\footnotetext{
${ }^{1} \mathrm{Li}$, Song, and Witt (2004) is excluded from Table 2 due to different models considered in the comparison.
} 
TABLE 2

INTERRELATIONSHIPS BETWEEN ALTERNATIVE DESTINATIONS WITH REGARD TO UK TOURISTS

\begin{tabular}{|c|c|c|c|c|c|c|c|c|c|c|c|c|c|}
\hline & France & Cyprus & Greece & Italy & Malta & Portugal & Spain & Turkey & Yugoslavia & Australia & New Zealand & Canada & US \\
\hline France & & & -D C C' & $-\mathrm{D}-\mathrm{C} \mathrm{C}^{\prime}$ & & A D C - C' & A D C C' & & & & & $-\mathrm{D}$ & $-\mathrm{D}$ \\
\hline Cyprus & & & & & $\mathrm{E}$ & & $\mathrm{E}$ & & & & & & \\
\hline Greece & -D C C' & & & -G -F -D -C -C', & & G F -D - C C & G F -D C - C' & $-G \mathbf{F}$ & $-F$ & & & $\mathrm{D}$ & $\mathrm{D}$ \\
\hline Italy & -D -C C' & & -G -D -F -C -C' & & & -G F -D C C' & G F -D -C C' & F G & $-F$ & & & $\mathrm{D}$ & $\mathrm{D}$ \\
\hline Malta & & $-E$ & & & & & $\mathrm{E}$ & & & & & & \\
\hline Portugal & A C C' D & & G - $C^{\prime} C^{\prime}-D$ F & F -G C C'-D & & $-\mathbf{C}$ & G F A -C - C' -D & $-G \mathbf{F}$ & $-F$ & & & D & D \\
\hline Spain & A C C' D & $\mathrm{E}$ & F G C - C' -D & F G -C C' -D & $\mathrm{E}$ & G A -C - $C^{\prime}-\mathbf{D} \mathbf{F}$ & & $-G F$ & $\mathrm{~F}$ & & & D & D \\
\hline Turkey & & & $\mathbf{F}-\mathrm{G}$ & F G & & F - G & F - G & & $-F$ & & & & \\
\hline Yugoslavia & & & $-F$ & $-F$ & & $-F$ & & $-F$ & & & & & \\
\hline Australia & & & & & & & & & & & $\mathbf{B}$ & & B \\
\hline New Zealand & & & & & & & & & & B & & & B \\
\hline Canada & $-\mathrm{D}$ & & $\mathrm{D}$ & $\mathrm{D}$ & & D & D & & & & & & $-D$ \\
\hline US & $-\mathrm{D}$ & & $\mathrm{D}$ & $\mathrm{D}$ & & D & D & & & B & B & $-\mathrm{D}$ & \\
\hline
\end{tabular}


TABLE 3

RANKINGS OF FORECASTING ACCURACY COMPARISON

\begin{tabular}{|c|c|c|c|c|c|c|c|c|c|c|c|c|c|c|c|c|c|c|c|c|c|c|c|c|c|c|c|c|c|}
\hline Study & 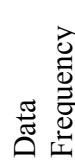 & 离 & 胥 & 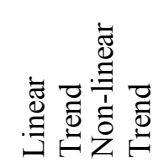 & $\begin{array}{l}\text { N } \\
\text { D. } \\
\text { : } \\
0 \\
0 \\
0\end{array}$ & 莺 & 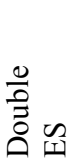 & 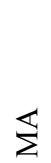 & $\overrightarrow{\frac{\alpha}{4}}$ & 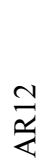 & 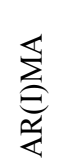 & 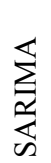 & 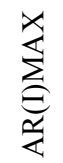 & $\sum_{\bigcup}$ & $\sum_{i}$ & 贡 & 台 & $\sum_{\infty}^{\infty}$ & $\begin{array}{l}\sum_{n} \\
\qquad \\
\qquad \\
\infty\end{array}$ & 䓀 & $\underset{Z}{\stackrel{3}{Z}}$ & $\sum_{\substack{\text { 是 } \\
\sum_{1}}}$ & $\begin{array}{l}\sum_{\substack{1 \\
1 \\
3}}^{n} \\
\sum_{3}\end{array}$ & $\sum_{\substack{1 \\
\vdots \\
j}}$ & 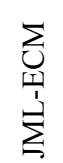 & $\underset{>}{\stackrel{2}{>}}$ & $\stackrel{z}{z}$ & 苛毠总 & 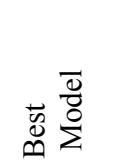 \\
\hline$\overline{\text { Akal (2004) }}$ & $\mathrm{A}$ & & & & & & & & & & & & & & & & & & & & & & & & & & & MAPE & \\
\hline Overall & & & & & & & & & & & & & 1 & & & & & & & 2 & & & & & & & & & ARMAX \\
\hline Cho (2001) & Q & & & & & & & & & & & & & & & & & & & & & & & & & & & MAPE & \\
\hline 1-8 steps ahead & & & & & & 3 & 4 & & & & & 1 & 2 & & & & & & & & & & & & & & & & SARIMA \\
\hline González \& Moral (1995) & $M$ & & & & & & & & & & & & & & & & & & & & & & & & & & & RMSE & \\
\hline 1 step ahead & & & & & & & & & & & & 3 & & & 1 & & & & 2 & & & & & 4 & & & & & TFM \\
\hline González \& Moral (1996) & $\mathrm{M}$ & & & & & & & & & & & & & & & & & & & & & & & & & & & RMSE & \\
\hline 1 step ahead & & & & 3 & & & & & & & & 2 & & & & & & & 1 & & & & & & & & & & STSM \\
\hline Kim \& Song (1998) & A & & & & & & & & & & & & & & & & & & & & & & & & & & & MAE & \\
\hline 3 steps ahead & & 6 & & & & 4 & & 5 & 3 & & 1 & & & & & & & & & & & & & & 2 & 7 & & & \\
\hline 5 steps ahead & & 7 & & & & 5 & & 6 & 4 & & 1 & & & & & & & & & & & & & & 3 & 2 & & & \\
\hline 7 steps ahead & & 7 & & & & 5 & & 6 & 3 & & 2 & & & & & & & & & & & & & & 1 & 4 & & & \\
\hline 10 steps ahead & & 6 & & & & 4 & & 5 & 3 & & 1 & & & & & & & & & & & & & & 2 & 7 & & & \\
\hline Overall & & 7 & & & & 4 & & 5 & 3 & & 1 & & & & & & & & & & & & & & 2 & 6 & & & ARMA \\
\hline Kulendran \& King (1997) & $\mathrm{Q}$ & & & & & & & & & & & & & & & & & & & & & & & & & & & MAPE & \\
\hline 1 step ahead & & & & & & & & & 3 & 5 & 3 & 1 & & & & & & & 2 & & & & & & 6 & & & & \\
\hline 2 steps ahead & & & & & & & & & 3 & 6 & 3 & 1 & & & & & & & 2 & & & & & & 3 & & & & \\
\hline 4 steps ahead & & & & & & & & & 1 & 6 & 3 & 2 & & & & & & & 5 & & & & & & 3 & & & & \\
\hline 8 steps ahead & & & & & & & & & 1 & 6 & 2 & 4 & & & & & & & 5 & & & & & & 3 & & & & \\
\hline Overall & & & & & & & & & 2 & 6 & 3 & 1 & & & & & & & 4 & & & & & & 5 & & & & SARIMA \\
\hline Kulendran \& Wilson (2000) & Q & & & & & & & & & & & & & & & & & & & & & & & & & & & MAPE & \\
\hline 1step ahead & & 2 & & & & & & & & & & 3 & & & & & & & & & & & & 1 & & & & & EG-ECM \\
\hline Law (2000) & A & & & & & & & & & & & & & & & & & & & & & & & & & & & MAPE & \\
\hline 1 step ahead & & 3 & & & & & 2 & 4 & & & & & & & & 5 & 1 & & & 6 & & & & & & & & & BNN \\
\hline Kulendran \& Witt (2001) & Q & & & & & & & & & & & & & & & & & & & & & & & & & & & MAPE & \\
\hline 1 step ahead & & 3 & & & & & & & & & 5 & 1 & & & & & & & 2 & & & & & & 4 & & & & \\
\hline 2 steps ahead & & 1 & & & & & & & & & 5 & 3 & & & & & & & 2 & & & & & & 4 & & & & \\
\hline 4 steps ahead & & 1 & & & & & & & & & 5 & 3 & & & & & & & 2 & & & & & & 4 & & & & \\
\hline 8 steps ahead & & 1 & & & & & & & & & 2 & 5 & & & & & & & 4 & & & & & & 3 & & & & \\
\hline Overall & & 1 & & & & & & & & & 5 & 4 & & & & & & & 2 & & & & & & 3 & & & & Naïve 1 \\
\hline Kulendran \& Witt (2003a) & Q & & & & & & & & & & & & & & & & & & & & & & & & & & & MAPE & \\
\hline 1 step ahead & & 6 & & & & & & & 5 & & 1 & 7 & & & & & & 2 & 4 & & & & & & 3 & & & & \\
\hline
\end{tabular}




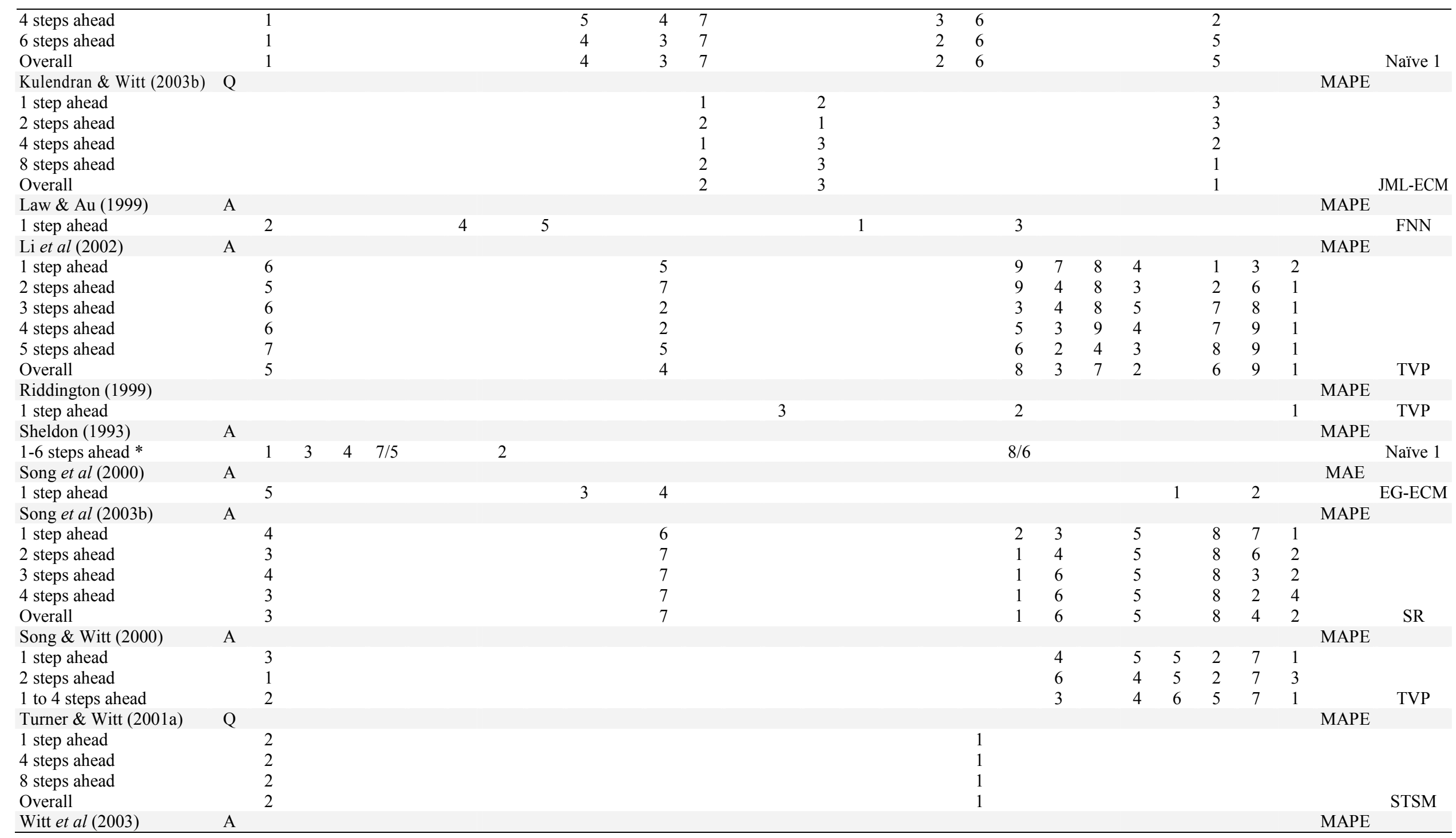




\begin{tabular}{|c|c|c|c|c|c|c|c|c|c|c|c|c|c|c|c|}
\hline 1 step ahead & & 3 & & & & & 4 & 5 & 1 & 6 & 8 & 7 & 2 & & \\
\hline 2 steps ahead & & 3 & & & & & 4 & 7 & 2 & 5 & 8 & 1 & 6 & & \\
\hline 3 steps ahead & & 3 & & & & & 4 & 5 & 2 & 7 & 8 & 1 & 6 & & \\
\hline Overall & & 3 & & & & & 4 & 6 & 1 & 7 & 8 & 2 & 5 & & ADLM \\
\hline Witt \& Witt $(1991,1992)$ & A & & & & & & & & & & & \multicolumn{4}{|c|}{ MAPE } \\
\hline 1 year ahead & & 1 & 4 & 7 & 6 & 2 & 3 & 5 & & & & & & & \\
\hline 2 years ahead & & 2 & 7 & 6 & 5 & 3 & 1 & 4 & & & & & & & \\
\hline Overall & & 2 & 5 & 7 & 6 & 3 & 1 & 4 & & & & & & & AR1 \\
\hline
\end{tabular}

Note: * 7/5 refer to the ranks of log quadratic and exponential trend fitting models, respectively, and 8/6 refer to linear and log-linear regressions, respectively.

TABLE 4

DESCRIPTIVE STATISTICS OF RANKINGS IN TABLE 3

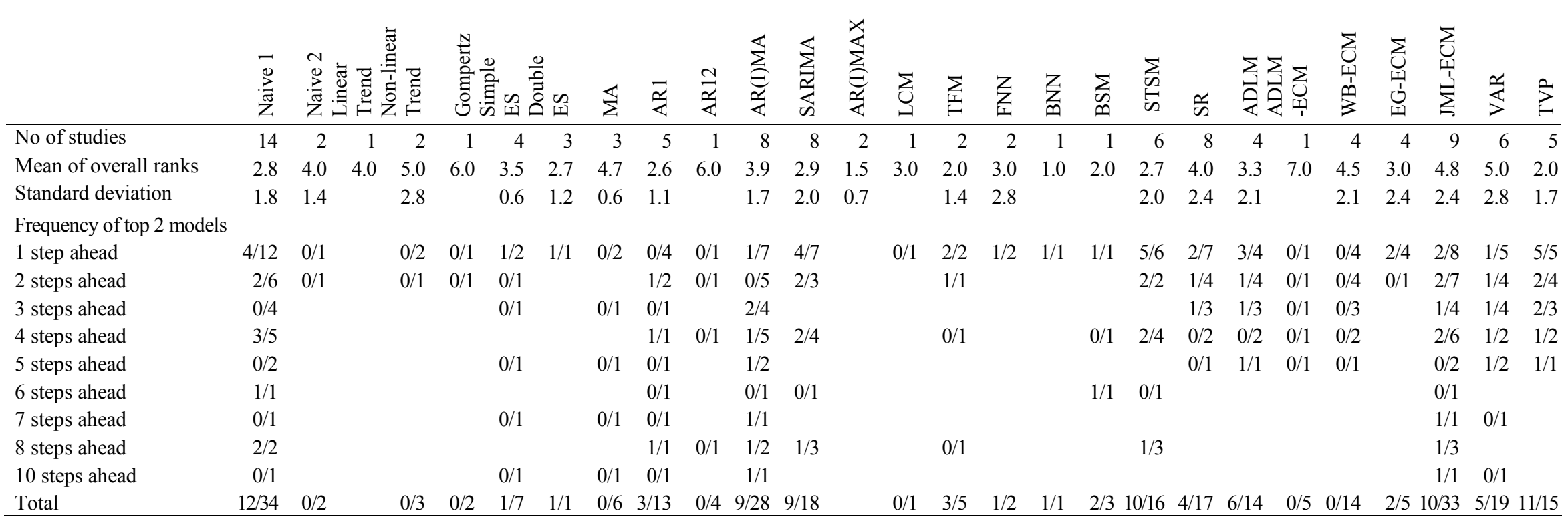




\section{Overall Performance of Forecasting Models}

Based on the rankings shown in Table 3, Table 4 provides some descriptive statistics. In the forecasting performance comparison, the naïve 1 model, which is also known as "no change" model, is often used as a benchmark. Various ECMs, especially the JML-ECM, are most often considered in the econometric forecasting of tourism demand. The static regression model also frequently appeared in the comparison as either a benchmark for econometric models or a competitor for timeseries models. Within the time-series forecasting scope, AR(I)MA models, including the seasonal version SAR(I)MA, has been the most popular.

The frequency with which each model appeared in the top two positions across all forecasting horizons suggests that the TVP model and the STSM, based on the same modeling technique, both performed relatively well in general. In particular, the TVP model was ranked number one in 7 and number two in 4 out of 15 cases. These findings are confirmed by the calculated means of overall ranks in all studies. On the other hand, the standard deviation of the overall ranks shows that the VAR model performed the least consistently, and its ranks varied from the top to the bottom. It should be noted that, due to the small number of studies being reviewed, some caution should be given to the interpretation of the means and standard deviations of the overall ranks.

\section{Factors Influencing Relative Forecasting Performance}

Tables 3 and 4 show that there is no one forecasting model that outperforms the others in all situations. Various factors are attributed to the discrepancies in performance between the studies.

Measures of Forecasting Error Magnitudes. Different measures for forecasting error magnitudes have been available for tourism demand forecasting evaluations. The predominant measure was MAPE, commonly used in all studies with only 4 exceptions and 144 out of 180 individual comparisons (from original papers). It was followed by RMSE and RMSPE, and they were used in 97 and 86 comparisons, respectively. In very few studies, other evaluation measures were applied, such as MAE and Theil's U statistic, acceptable output percentage $(Z)$ and normalized correlation coefficient $(r)$. Comparing different measures of the relative forecasting performance of the estimated models, the MAPE and RMSE (or RMSPE) gave the same rankings in only 32 out of 117 cases. The discrepancy was evident especially when large variations appeared among individual forecast errors. The inconsistency between the rankings given by two groups of measures is due to different assumptions regarding the forms of the loss functions. The MAPE is associated with a linear loss function, while the RMSE and RMSPE are consistent with the notion of a quadratic form (Theil 1966). As a result, the RMSE and RMSPE are more sensitive to one extremely bad forecast.

It should be noted that the above measures such as MAPE and RMSPE do not have a statistical underpinning. To examine if the difference in the accuracy of competing forecasts is significant, formal statistical tests need to be performed. So far, only Witt, Song, and Louvieris (2003) have examined forecasting bias and directional change forecasting performance using formal statistics in a tourism context. Such formal tests should be given more attention in forecasting performance comparisons. 
Time-Horizons of Forecasting. The forecasting power varies across different forecasting horizons. In general, due to increasing uncertainty, the longer the forecasting horizon, the less accurate the predictions. The frequency that each model was ranked in the top two at each forecasting horizon (Table 4) suggests that the TVP model and the STSM outperformed their competitors especially as far as short-run (one-step-ahead) forecasting was concerned. Witt, Song, and Louvieris (2003) also showed that the TVP model provided sound performance in one-step-ahead directional change forecasts. Frequencies displayed in Table 4 also show that the JML-ECM forecast more accurately in the medium to long run (5-10-steps ahead) than in the short run. So far the ADLM-ECM and the WB-ECM have not shown satisfactory performance in spite of considering different forecasting horizons. However, due to extremely few applications of these models, general conclusions cannot be drawn, unless more tests of their forecasting abilities are undertaken in the future research. It should be noted that, due to the rationale and model specification, the ECMs are likely to perform better where the differenced demand variable (or the growth rate in the log-transform model) is concerned. However, no published study has conducted this empirical test yet.

Data Frequency. Annual data were used most frequently in the above forecasting exercises (by 13 studies), followed by quarterly data (7 studies) and monthly data (only 2 studies). Monthly and quarterly data possess different properties compared with annual data, because tourism demand exhibits strong seasonality. Therefore, the models that readily account for seasonal variations, such as the SAR(I)MA model and the STSM, are preferable where these types of data are used. They generally performed well, except in Kulendran and Witt (2003a). Most of the other econometric models were most often related to annual data except the JML-ECM, which was used often for both annual and quarterly data and performed slightly better where quarterly data were utilized. In the future it will be worth testing the forecasting abilities of other advanced econometric models in dealing with seasonality in tourism demand. In particular, since the STSM and the TVP model both have shown their superior performance when seasonal and annual data were concerned, respectively, the combination of these two models and the application to seasonal data is likely to be advantageous.

Forecasting Competitors. The relative forecasting performance, in terms of the rank of an evaluated model, to a certain extent depends on which competitors take part in the comparison. For example, in the comparisons where the static regression model was the only causal model (Law 2000; Law and Au 1999; Witt and Witt 1991 and 1992), it was always outperformed by time series models. In particular, all the results of the one-year-ahead forecasting of Witt and Witt (1992) showed that the naive 1 model was superior to all the other non-causal and causal candidates. However, in the forecasting comparisons involving more advanced causal models, non-causal models did not show outstanding performance, especially when annual data were used. The latter outperformed causal models and were ranked top in only 6 out of 16 studies in terms of the overall ranking, 4 being associated with quarterly data. In particular, the naive 1 model generated the best forecasts only in 3 out of 12 overall evaluations and 6 out of 32 comparisons at individual forecast horizons. These results suggest that applying advanced econometric techniques to tourism demand forecasting should be encouraged, especially in the cases where annual data are to be used.

Data Generation Processes (DGPs). Within a single study where the same models are applied for different origin-destination pairs, their performance may vary from 
case to case. An extreme example can be seen in the results of $\mathrm{Li}$, Song, and Witt (2002), where the ARIMA model was shown to generate the most accurate forecasts in the cases of Japan and Singapore, while the second poorest for Australia and the US. Furthermore, the WB-ECM outperforms all the other candidates in Australia's case, but is ranked the last second in the UK's case. Similar phenomena can also be seen in Kim and Song (1998), Kulendran and King (1997), Kulendran and Wilson (2000) and so on. Such discrepancies in models' performance across different countries may well result from different DGPs relating to these destinations or origins, especially in the cases where destination- or origin-specific one-off events take place.

A model's ability to capture the intervention effect on the time series may also affect predictive accuracy. Within the tourism context, however, the impact of interventions, as well as outlier detection, has only been assessed for timeseries models (see, for instance, Goh and Law 2002; Chu 2004). No study has examined the effects of interventions or outliers on the forecasting performance of econometric models.

\section{Emerging Forecasting Models}

The forecasting performance of the AR(I)MAX model and the error correction LAIDS model has been examined in the tourism context recently. Cho (2001) compared the forecasting accuracy of the ARIMAX model with the ARIMA and two exponential smoothing models. The results show that the ARIMAX model was always ranked in the top two and first place in 2 out of 6 cases. Akal (2004) found the ARMA model outperformed the static regression model in his research. However, none of the advanced econometric models entered the competitions in these two studies, and it should be of interest to fill this gap in further studies. The study of $\mathrm{Li}$, Song, and Witt (2004) is the only one that compared forecasting accuracy amongst demand system models. Forecasts of tourism demand measured by market shares have particular importance for competitive analysis between competing destinations. Li, Song, and Witt (2004) study showed that by incorporating the ECM into the LAIDS specification, its short-run (one-year-ahead) forecasting accuracy of market shares improved remarkably. However, no longer time horizons have been considered, and the performance in forecasting market share changes has not been investigated yet. These indicate possible directions for further studies on tourism demand forecasting.

\section{SUMMARY}

The continuing growth of international tourism demand in the last decade has stimulated studies in this field. To reflect the latest developments in the research on econometric modeling and forecasting of tourism demand, this study provides an upto-date survey of 84 empirical studies in this area, mostly published after 1990. Compared to the studies between the 1960s and 1980s, more advanced econometric techniques, such as the CI/ECM, VAR, TVP and AIDS models have been applied to tourism demand studies in the 1990s and early 2000s. These methods contribute to improvements in the understanding of international tourism demand. In particular, the $\mathrm{CI} / \mathrm{ECM}$ approaches identify the differences between the long-run and short-run demand elasticities, and the TVP model demonstrates the evolution of elasticities over time. A further review of 23 tourism demand forecasting studies suggests that there is no single model that outperforms the others in all cases. The performance of alternative models is situation-specific, and many factors may influence their 
forecasting accuracy. In general, the TVP model and the STSM perform relatively well, especially for short-run forecasting. Where advanced econometric models compete with their univariate time-series counterparts or the conventional benchmark no-change model, the econometric models tend to outperform the others, especially as far as annual data are concerned.

Some emerging models have shown advantages in modeling and forecasting international tourism demand. Broader applications and further improvements of these methodologies are likely to benefit research in this area. In particular, the following directions are of interest and value in future econometric studies of tourism demand.

1. Further application of the AIDS/LAIDS especially its ECM form for analyzing and predicting market shares and their variations.

2. Combination of the STSM and TVP model to forecast seasonal tourism demand.

3. Further employment of the AR(I)MAX model and examination of its forecasting performance in comparison with other econometric models.

4. Comparison of the abilities of alternative models to forecast tourism demand changes (or growth).

5. Investigation of the forecasting performance of advanced econometric models in dealing with seasonality in tourism demand.

In view of the diversity of research findings, including those derived from newly emerging techniques which has resulted in a relatively small number of observations in this survey (especially those related to forecasting comparison), caution should be exercised in interpreting the generalized findings of this paper.

\section{REFERENCES}

Akal, M. (2004). "Forecasting Turkey's Tourism Revenues by ARMAX Model." Tourism Management, 25: 565-80.

Akis, S. (1998). "A Compact Econometric Model of Tourism Demand for Turkey." Tourism Management, 19: 99-102.

Ashworth, J. and P. Johnson (1990). "Holiday Tourism Expenditure: Some Preliminary Econometric Results." The Tourist Review, 3: 12-19.

Bakkal, I. (1991). "Characteristics of West German Demand for International Tourism in the Northern Mediterranean Region.” Applied Economics, 23:295-304.

Cho, V. (2001). "Tourism Forecasting and Its Relationship with Leading Economic Indicators." Journal of Hospitality and Tourism Research, 25(4): 399-420.

Chu, F. (2004). "Forecasting Tourism Demand: A Cubic Polynomial Approach." Tourism Management, 25: 209-218.

Crouch, G. I. (1992). "Effect of Income and Price on International Tourism." Annals of Tourism Research, 19: 643-64.

(1994a). "Demand Elasticities for Short-Haul versus Long-Haul Tourism." Journal of Travel Research, 33: 2-7.

(1994b). "The Study of International Tourism Demand: A Review of Findings." Journal of Travel Research, 33: 12-23. 
_ (1994c). "The Study of International Tourism Demand: A Survey of Practice.” Journal of Travel Research, 33: 41-54.

(1995). "A Meta-Analysis of Tourism Demand.” Annals of Tourism Research, 22: 10318.

(1996). "Demand Elasticities in International Marketing: A Meta-Analytical Application to Tourism." Journal of Business Research, 36: 117-36.

De Mello, M., A. Pack and M. T. Sinclair (2002). "A System of Equations Model of UK Tourism Demand in Neighbouring Countries." Applied Economics, 34: 509-521.

Deaton, A. S. and J. Muellbauer (1980). “An Almost Ideal Demand System.” American Economic Review, 70: 312-26.

Di Matteo, L. (1999). "Using Alternative Methods to Estimate the Determinants of CrossBorder Trips.” Applied Economics, 31: 77-88.

Di Matteo, L. and R. Di Matteo (1993). "The Determinants of Expenditures by Canadian Visitors to the United States." Journal of Travel Research, 31: 34-42.

Divisekera, S. (2003). "A Model of Demand for International Tourism." Annals of Tourism Research, 30: 31-49.

Dritsakis, N. (2004). "Cointegration Analysis of German and British Tourism Demand for Greece.” Tourism Management, 25: 111-19.

Dritsakis, N. and J. Papanastasiou (1998). "An Econometric Investigation of Greek Tourism: A Note." Journal for Studies in Economics and Econometrics, 22: 115-22.

Dritsakis, N. and S. Athanasiadis (2000). "An Econometric Model of Tourist Demand: The Case of Greece." Journal of Hospitality and Leisure Marketing, 7: 39-49.

Durbarry, R. and M. T. Sinclair (2003). "Market Shares Analysis: The Case of French Tourism Demand.” Annals of Tourism Research, 30: 927-41.

Engle, R. F. and C. W. J. Granger (1987). "Cointegration and Error Correction: Representation, Estimation and Testing." Econometrica, 55: 251-76.

Friedman, M. (1956). A Theory of the Consumption Function. Princeton University Press.

Fujii, E., M. Khaled and J. Mark (1985). "An Almost Ideal Demand System for Visitor Expenditures." Journal of Transport Economics and Policy, 19: 161-71.

Gallet, C. A. and B. M. Braun (2001). "Gradual Switching Regression Estimates of Tourism Demand." Annals of Tourism Research, 28: 503-08.

García-Ferrer, A. and R. Queralt (1997). "A Note on Forecasting International Tourism Demand in Spain.” International Journal of Forecasting, 13: 539-49.

Gerakis, A. S. (1965). "Effects of Exchange-Rate Devaluations and Revaluations on Receipts from Tourism." International Monetary Fund Staff Papers, 12: 365-84.

Goh, C. and R. Law (2002). "Modeling and Forecasting Tourism Demand for Arrivals with Stochastic Nonstationary Seasonality and Intervention." Tourism Management, 23: 499510 .

González, P. and P. Moral (1995). "An Analysis of the International Tourism Demand in Spain." International Journal of Forecasting, 11: 233-51.

(1996). "Analysis of Tourism Trends in Spain." Annals of Tourism Research, 23: 73954.

Gray, H. P. (1966). "The Demand for International Travel by United States and Canada." International Economic Review, 7: 83-92.

Greenidge, K. (2001). "Forecasting Tourism Demand: An STM Approach." Annals of Tourism Research, 28: 98-112. 
Guthrie, H. W. (1961). "Demand for Tourists' Goods and Services in a World Market." Papers and Proceedings of the Regional Science Association, 7: 159-75.

Harris, R. and R. Sollis (2003). Applied Tme Series Modeling and Forecasting. Wiley: Chichester.

Hendry, D. F. (1995). Dynamics Economics: Advanced Text in Econometrics. Oxford University Press: Oxford.

Holmes, R. A. and, A. F. M. Shamsuddin (1997). "Short- and Long-Term Effects of World Exposition 1986 on US Demand for British Columbia Tourism.” Tourism Economics, 3: 137-60.

Icoz, O., T. Var, and, M. Kozaka (1998). "Tourism Demand in Turkey.” Annals of Tourism Research, 25: 236-40.

Ismail, J. A., T. J. Iverson and, L. A. Cai (2000). "Forecasting Japanese Arrivals to Guam: An Empirical Model." Journal of Hospitality and Leisure Marketing.27: 51-63.

Jensen, T. C. (1998). "Income and Price Elasticities by Nationality for Tourists in Denmark." Tourism Economics, 4: 101-30.

Johansen, S. (1988). "A Statistical Analysis of Cointegration Vectors." Journal of Economic Dynamics and Control, 12: 231-54.

Jørgensen, F. and, G. Solvoll (1996). "Demand Models for Inclusive Tour Charter: The Norwegian Case.” Tourism Management, 17: 17-24.

Kim, S. and H. Song (1998). "Analysis of Tourism Demand in South Korea: A Cointegration and Error Correction Approach." Tourism Analysis, 3: 25-41.

Kim, Y. and, M. Uysal (1998). "Time-Dependent Analysis for International Hotel Demand in Seoul.” Tourism Economics, 4: 253-63.

Kulendran, N. (1996). "Modelling Quarterly Tourism Flows to Australia." Tourism Economics, 2: 203-22.

Kulendran, N. and K. Wilson (2000). "Modelling Business Travel.” Tourism Economics, 6: 47-59.

Kulendran, N. and M. King (1997). "Forecasting International Quarterly Tourism Flows Using Error Correction and Time Series Models." International Journal of Forecasting, 13: 319-27.

Kulendran, N. and S. F. Witt (2001). "Cointegration versus Least Squares Regression." Annals of Tourism Research, 28: 291-311.

(2003a). "Forecasting the Demand for International Business Tourism." Journal of Travel Research, 41: 265-71.

(2003b). "Leading Indicator Tourism Forecasts." Tourism Management, 24: 503-10.

Lanza, A. P. Temple and G. Urga (2003). "The Implications of Tourism Specialisation in the Long Run: An Econometric Analysis for 13 OECD Economies." Tourism Management, 24: $315-21$.

Lathiras, P. and, C. Siriopoulos (1998). "The Demand for Tourism to Greece: A Cointegration Approach.” Tourism Economics, 4: 171-85.

Law, R. (2000). "Back-propagation Learning in Improving the Accuracy of Neural Networkbased Tourism Demand Forecasting." Tourism Management, 21: 331-40.

Law, R. and N. Au (1999). "A Neural Network Model to Forecast Japanese Demand for Travel to Hong Kong." Tourism Management, 20: 89-97.

Ledesma-Rodríguez, F. J., M. Navarro-Ibánez and J. V. Pérez-Rodríguez (2001). "Panel Data and Tourism: A Case Study of Tenerife." Tourism Economics, 7: 75-88.

Lee, C. K., T. Var and T. W. Blaine (1996). "Determinants of Inbound Tourism Expenditure." Annals of Tourism Research, 23: 527-42. 
Li, G., H. Song and S. F. Witt (2002). "Econometric Analysis and Forecasts of International Tourism Demand in Thailand." Paper presented at the Fifth Biennial Conference on Tourism in Asia: Development, Marketing and Sustainability, Hong Kong, May 23-25, 2002 .

_ (2004). "Modelling Tourism Demand: A Dynamic Linear AIDS Approach.” Journal of Travel Research, 43: 141-150.

Lim, C. (1997a). "An Econometric Classification and Review of International Tourism Demand Models." Tourism Economics, 3: 69-81.

_ (1997b). "Review of International Tourism Demand Models." Annals of Tourism Research, 24: 835-49.

_ (1999). “A Meta-Analytic Review of International Tourism Demand.” Journal of Travel Research, 37: 273-84.

Lim, C. and M. McAleer (2001). "Cointegration Analysis of Quarterly Tourism Demand by Hong Kong and Singapore for Australia." Applied Economics, 33: 1599-619.

(2002). "Time Series Forecasts of International Travel Demand for Australia." Tourism Management, 23: 389-96.

Lyssiotou, P. (2001). "Dynamic Analysis of British Demand for Tourism Abroad.” Empirical Economics, 15: 421-36.

Mangion, M. L., R. Durbarry and M. T. Sinclair (2003). "AIDS and Hedonic Pricing Models: Their Relevance to Tourism Practitioners." Paper presented at the Tourism Modelling and Competitiveness Conference, Paphos, Cyprus.

Morley, C. L. (1997). "An Evaluation of the Use of Ordinary Least Squares for Estimating Tourism Demand Model.” Journal of Travel Research, 35: 69-73.

(1998). “A Dynamic International Demand Model.” Annals of Tourism Research, 25: 70-84.

Morris, A., K. Wilson and, S. Bakalis (1995). "Modelling Tourism Flows from Europe to Australia." Tourism Economics, 1: 147-67.

O'Hagan, J. W. and M. J. Harrison (1984). "Market Shares of US Tourism Expenditure in Europe: An Econometric Analysis." Applied Economics, 16: 919-31.

Papatheodorou, A. (1999). "The Demand for International Tourism in the Mediterranean Region." Applied Economics, 31: 619-30.

Payne, J. E. and A. Mervar (2002). "A Note on Modelling Tourism Revenues in Croatia." Tourism Economics, 8:103-09.

Pesaran, M. H. and Y. Shin (1995). "An Autoregressive Distributed Lag Modelling Approach to Cointegration Analysis.” In Strom, S., A. Holly and P. Diamond, eds., Centennial Vol. of Rangar Frisch, Econometric Society Monograph. Cambridge University Press: Cambridge.

Pyo, S., M. Uysal and R. McLellan (1991). "A Linear Expenditure Model for Tourism Demand." Annals of Tourism Research, 31: 619-30.

Qiu, H. and J. Zhang (1995). "Determinants of Tourist Arrivals and Expenditures in Canada." Journal of Travel Research, 34: 43-49.

Qu, H. and, S. Lam (1997). "A Travel Demand Model for Mainland Chinese Tourists to Hong Kong.” Tourism Management, 18: 593-97.

Riddington, G. (1999). "Forecasting Ski Demand: Comparing Learning Curve and Time Varying Parameter Approaches." Journal of Forecasting, 18: 205-14.

Rossello-Nadal, J. (2001). "Forecasting Turning Points in International Visitor Arrivals in the Balearic Islands.” Tourism Economics, 7: 365-80. 
Rosselló-Nadal, J., A. Riera-Font and A. Sansó-Rosselló (2004). "The Economic Determinants of Seasonal Patterns." Annals of Tourism Research, 31: 697-711.

Shan, J. and, K. Wilson (2001). "Causality between Trade and Tourism: Empirical Evidence from China." Applied Economics Letters, 8: 279-83.

Sheldon, P. J. (1993). "Forecasting Tourism: Expenditure versus Arrivals." Journal of Travel Research, 32: 13-20.

Sheldon, P. J. and T. Var (1985). "Tourism Forecasting: A Review of Empirical Research." Journal of Forecasting, 4: 183-95.

Smeral, E. and S. Witt (1996). "Econometric Forecasts of Tourism Demand to 2005." Annals of Tourism Research, 23: 891-907.

Smeral, E. and, A. Weber (2000). "Forecasting International Tourism Trends to 2010." Annals of Tourism Research, 27: 982-1006.

Smeral, E., S. F. Witt and C. A. Witt (1992). "Econometric Forecasts: Tourism Trends to 2000." Annals of Tourism Research, 29: 450-66.

Song, H. and K. F. Wong (2003). "Tourism Demand Modeling: A Time-Varying Parameter Approach." Journal of Travel Research, 42: 57-64.

Song, H., K. F. Wong and K. S. Chon (2003). "Modelling and Forecasting the Demand for Hong Kong Tourism." International Journal of Hospitality Management, 22: 435-51.

Song, H., P. Romilly and X. Liu (2000). "An Empirical Study of Outbound Tourism Demand in the U.K.." Applied Economics, 32: 611-24.

Song, H., S. F. Witt and G. Li (2003). "Modelling and Forecasting the Demand for Thai Tourism." Tourism Economics, 9: 363-87.

Song, H., S. F. Witt and T. C. Jensen (2003). "Tourism Forecasting: Accuracy of Alternative Econometric Models.” International Journal of Forecasting, 19: 123-41.

Stone, J. R. N. (1954). "Linear Expenditure Systems and Demand Analysis: An Application to the Pattern of British Demand." Economic Journal, 64: 511-27.

Syriopoulos, T. (1995). "A Dynamic Model of Demand for Mediterranean Tourism." International Review of Applied Economics, 9: 318-36.

Syripopoulos, T. and T. Sinclair (1993). "A Dynamic Model of Demand for Mediterranean Countries." Applied Economics, 25: 1541-52.

Theil, H. (1966). Applied Economic Forecasting. North-Holland: Amsterdam.

Turner, L. W. and S. F. Witt (2001a) "Factors Influencing Demand for International Tourism: Tourism Demand Analysis Using Structural Equation Modelling, Revisited." Tourism Economics, 7: 21-38.

(2001b). "Forecasting Tourism Using Univariate and Multivariate Structural Time Series Models." Tourism Economics, 7: 135-47.

Turner, L. W., Y. Reisinger and S. F. Witt (1998). "Tourism Demand Analysis Using Structural Equation Modelling." Tourism Economics, 4: 301-23.

Uysal, M. and J. L. Crompton (1985). "An Overview of Approaches Used to Forecast Tourism Demand." Journal of Travel Research, 24: 7-15.

Uysal, M. and M. Roubi (1999). "Artificial Neural Networks versus Multiple Regression in Tourism Demand Analysis." Journal of Travel Research, 38: 111-18.

Vanegas, M., Sr and R. R. Croes (2000). "Evaluation of Demand: US Tourists to Aruba." Annals of Tourism Research, 27: 946-63.

Var, T. and G. M. Icoz (1990). "Factors Affecting International Tourism Demand for Turkey." Annals of Tourism Research, 17: 606-10. 
Vogt, M. G. and C. Wittayakorn (1998). "Determinants of the Demand for Thailand's Exports of Tourism.” Applied Economics, 30: 711-15.

White, K. J. (1985). "An International Travel Demand Model: US Travel to Western Europe.” Annals of Tourism Research, 12: 529-45.

Wickens, M. R. and T. S. Breusch (1988). "Dynamic Specification, the Long-Run and the Estimation of Transformed Regression Model." Economic Journal, 98 (Conference): 189-205.

Witt, C. A. and S. F. Witt (1990) “Appraising an Econometric Forecasting Model.” Journal of Travel Research, 28: 30-34.

Witt, S. F. and C. A. Witt (1991) "Tourism Forecasting: Error Magnitude, Direction of Change Error and Trend Change Error." Journal of Travel Research, 30: 26-33.

(1992). Modeling and Forecasting Demand in Tourism. Academic Press: London.

_ (1995). "Forecasting Tourism Demand: A Review of Empirical Research." International Journal of Forecasting, 11: 447-75.

Witt, S.F., H. Song and P. Louvieris (2003). "Statistical Testing in Forecasting Model Selection." Journal of Travel Research, 42: 151-58.

Zellner, A. C. (1962). "An Efficient Method of Estimating Seemingly Unrelated Regressions and Test for Aggregation Bias." Journal of the American Statistical Association, 57: $348-68$ 